\title{
Optimal embeddings and compact embeddings of Bessel-potential-type spaces
}

\author{
Amiran Gogatishvili · Júlio S. Neves • Bohumír Opic
}

Received: 25 February 2007 / Accepted: 6 May 2008

C Springer-Verlag 2008

\begin{abstract}
First, we establish necessary and sufficient conditions for embeddings of Bessel potential spaces $H^{\sigma} X\left(\mathbb{R}^{n}\right)$ with order of smoothness less than one, modelled upon rearrangement invariant Banach function spaces $X\left(\mathbb{R}^{n}\right)$, into generalized Hölder spaces. To this end, we derive a sharp estimate of modulus of smoothness of the convolution of a function $f \in X\left(\mathbb{R}^{n}\right)$ with the Bessel potential kernel $g_{\sigma}, 0<\sigma<1$. Such an estimate states that if $g_{\sigma}$ belongs to the associate space of $X$, then

$$
\omega\left(f * g_{\sigma}, t\right) \precsim \int_{0}^{t^{n}} s^{\frac{\sigma}{n}-1} f^{*}(s) d s \text { for all } t \in(0,1) \text { and every } f \in X\left(\mathbb{R}^{n}\right) .
$$

Second, we characterize compact subsets of generalized Hölder spaces and then we derive necessary and sufficient conditions for compact embeddings of Bessel potential spaces $H^{\sigma} X\left(\mathbb{R}^{n}\right)$ into generalized Hölder spaces. We apply our results to the case when $X\left(\mathbb{R}^{n}\right)$ is the Lorentz-Karamata space $L_{p, q ; b}\left(\mathbb{R}^{n}\right)$. In particular, we are able to characterize optimal embeddings of Bessel potential spaces $H^{\sigma} L_{p, q ; b}\left(\mathbb{R}^{n}\right)$ into generalized Hölder spaces and also compact embeddings of spaces in question. Applications cover both superlimiting and limiting cases.
\end{abstract}

\footnotetext{
A. Gogatishvili · B. Opic

Mathematical Institute, Academy of Sciences of the Czech Republic, Žitná 25, 11567 Prague 1, Czech Republic

e-mail: gogatish@math.cas.cz

J. S. Neves $(\bowtie)$

CMUC, Department of Mathematics, University of Coimbra, Apartado 3008, 3001-454 Coimbra, Portugal

e-mail: jsn@mat.uc.pt

B. Opic

Department of Mathematics and Didactics of Mathematics, Technical University of Liberec, Hálkova 6, 46117 Liberec, Czech Republic

e-mail: opic@math.cas.cz
} 


\section{Introduction}

Classical Bessel potential spaces $H^{\sigma, p}\left(\mathbb{R}^{n}\right)=H^{\sigma} L^{p}\left(\mathbb{R}^{n}\right)$, introduced in [2] and [8], have played a significant role in mathematical analysis and in applications for many years (cf. [36,39], etc.). These spaces are modelled upon the scale of Lebesgue spaces $L^{p}\left(\mathbb{R}^{n}\right)$ and they coincide with the Sobolev spaces $W^{k, p}\left(\mathbb{R}^{n}\right)=W^{k} L^{p}\left(\mathbb{R}^{n}\right)$ when $\sigma=k \in \mathbb{N}$ and $p \in(1,+\infty)$. However, it has gradually become clear that to handle some situations (especially limiting ones) a more refined tuning is desirable. For this purpose, one needs to replace the Lebesgue scale of spaces by a scale of spaces which can be more finely tuned. For example, to obtain estimates of degenerate elliptic differential operators with coefficients having singular behaviour, Edmunds and Triebel (cf. $[18,19])$ replaced the $L^{p}$ spaces by the spaces $L^{p}(\log L)^{q}$ of Zygmund type. The same replacement enables one to obtain interesting results concerning smoothness properties of orientation-preserving maps (see [29] for references and an account of work in this direction).

In a series of recent papers [12-14,35] a systematic research of embeddings of Bessel potential spaces with order of smoothness $\sigma \geq 1$ modelled upon generalized LorentzZygmund (GLZ) spaces was carried out. The authors of those papers established embeddings of such spaces either into GLZ-spaces or into Hölder-type spaces $C^{0, \lambda(\cdot)}(\bar{\Omega})$ and showed that their results are sharp (within the given scale of target spaces) and fail to be compact. They also clarified the role of the logarithmic terms involved in the quasi-norms of the spaces mentioned. This role proved to be important especially in limiting cases. In particular, they obtained refinements of the Sobolev embedding theorems, Trudinger's limiting embedding as well as embeddings of Sobolev spaces into $\lambda(\cdot)$-Hölder continuous functions including the result of Brézis and Wainger about almost Lipschitz continuity of elements of the (fractional) Sobolev space $H^{1+n / p, p}\left(\mathbb{R}^{n}\right)$ (cf. [7]).

Although GLZ-spaces form an important scale of spaces containing, for example, Zygmund classes $L^{p}(\log L)^{\alpha}$, Orlicz spaces of multiple exponential type, Lorentz spaces $L^{p, q}$, Lebesgue spaces $L^{p}$, etc., GLZ-spaces are a particular case of more general spaces, namely the Lorentz-Karamata (LK) spaces.

The embeddings mentioned above were extended in [32,33] to the case when Besselpotential spaces are modelled upon LK-spaces. Since Neves considered more general targets (besides LK-spaces and Hölder-type spaces also generalized Hölder spaces), in several cases he obtained improvements of embeddings from [12-14]. The sharpness and the noncompactness of these embeddings were proved in [21,22]. (An account of the principal embedding results involving Bessel potential spaces modelled upon LK-spaces is also given in [11]).

Note that one of main steps in the proof of continuous embeddings of Bessel potential spaces with order of smoothness $\sigma \geq 1$ into Hölder-type spaces consists in the application of Stein's inequality (cf. [5, Exercise 12(b), p. 430] or [10]). This inequality states that a function $\mathrm{u}$, such that the norm of its distributional gradient $|\nabla u|$ belongs locally to the Lorentz space $L^{n, 1}\left(\mathbb{R}^{n}\right)$, can be redefined on a set of measure zero so that the modulus of smoothness $\omega(u, \cdot)$ of $u$ satisfies the inequality

$$
\omega(u, t) \precsim \int_{0}^{t^{n}} s^{\frac{1}{n}-1}|\nabla u|^{*}(s) d s \text { for all } t \in(0,1)
$$

(here $|\nabla u|^{*}$ denotes the non-increasing rearrangement of $|\nabla u|$ ).

In [15] and [16] the authors analysed the situation when the order of smoothness is less than one. In such a case one cannot use the method in which inequality (1) and a lifting 
argument (based on [13, Lemma 4.1] or [22, Lemma 4.5], which extend the Calderón result [8, Theorem 7]) are applied to reduce the superlimiting case to the sublimiting one, and a new approach was used. The authors of those papers established embeddings of such spaces into Hölder-type spaces $C^{0, \lambda(\cdot)}(\bar{\Omega})$ and showed that their results concerning nonlimiting cases are sharp (within the given scale of target spaces) and fail to be compact.

Here, we establish necessary and sufficient conditions for embeddings of Bessel potential spaces $H^{\sigma} X\left(\mathbb{R}^{n}\right)$ with order of smoothness less than one, modelled upon rearrangement invariant Banach function spaces $X\left(\mathbb{R}^{n}\right)$, into generalized Hölder spaces $\Lambda_{\infty, r}^{\lambda(\cdot)}(\bar{\Omega}), 0<r \leq+\infty$. (We refer to Sect. 2 for precise definitions. Note also that the space $\Lambda_{\infty, \infty}^{\lambda(\cdot)}(\bar{\Omega})$ coincides with the space $C^{0, \lambda(\cdot)}(\bar{\Omega})$ mentioned above.) For this purpose, we derive a convenient replacement of (1). Namely, if $\sigma \in(0,1), X=X\left(\mathbb{R}^{n}\right)$ is a rearrangement invariant Banach function space and the Bessel potential kernel $g_{\sigma}$ belongs to the associate space of $X$, then we prove that (cf. Theorem 1 below)

$$
\omega\left(f * g_{\sigma}, t\right) \precsim \int_{0}^{t^{n}} s^{\frac{\sigma}{n}-1} f^{*}(s) d s \text { for all } t \in(0,1) \text { and every } f \in X,
$$

where $f^{*}$ denotes the non-increasing rearrangement of $f$. Moreover, estimate (2) is sharp in the sense that

$$
\omega\left(\bar{f} * g_{\sigma}, t\right) \succsim \int_{0}^{t^{n}} s^{\frac{\sigma}{n}-1} f^{*}(s) d s \text { for all } t \in(0,1) \text { and every } f \in X,
$$

where

$$
\bar{f}(x)=f^{*}\left(\beta_{n}|x|^{n}\right) \chi\left\{y \in \mathbb{R}^{n}: y_{1}>0\right\} \cap B(0,1)(x), \quad x=\left(x_{1}, \ldots, x_{n}\right) \in \mathbb{R}^{n},
$$

and $\beta_{n}$ is the volume of the unit ball in $\mathbb{R}^{n}$. Inequalities (2) and (3) enable us to show that the continuous embedding of the Bessel potential space $H^{\sigma} X\left(\mathbb{R}^{n}\right)$ into the generalized Hölder space $\Lambda_{\infty, r}^{\mu(\cdot)}\left(\overline{\mathbb{R}^{n}}\right)$ is equivalent to the condition that

$$
g_{\sigma} \text { belongs to the associate space of } X
$$

and to the boundedness of the Hardy-type operator

$$
H: \bar{X} \longrightarrow L_{r}\left((0,1) ; t^{-1 / r}(\mu(t))^{-1}\right),
$$

where the operator $H$ is defined by

$$
(H f)(t):=\int_{0}^{t^{n}} s^{\frac{\sigma}{n}-1} f^{*}(s) d s
$$

$\bar{X}$ denotes the representation space of $X$ and $L_{r}\left((0,1) ; t^{-1 / r}(\mu(t))^{-1}\right)$ is the weighted Lebesgue space over the interval $(0,1)$ (cf. Corollary 2 below and the fact that $\|f\|_{X}=$ $\left.\left\|f^{*}\right\|_{\bar{X}}\right)$.

Furthermore, we characterize compact subsets of generalized Hölder spaces $\Lambda_{\infty, r}^{\mu(\cdot)}(\bar{\Omega})$, $0<r<+\infty$, with a bounded domain $\Omega$ in $\mathbb{R}^{n}$ (cf. Theorem 5 below) and then we derive necessary and sufficient conditions for compact embeddings of Bessel potential spaces $H^{\sigma} X\left(\mathbb{R}^{n}\right)$ into generalized Hölder spaces $\Lambda_{\infty, r}^{\mu(\cdot)}(\bar{\Omega}), 0<r<+\infty$. To this end, we make use of local versions of inequalities (2) and (3) [cf. (48) and (49) below] to show that the 
compactness of the embedding in question is equivalent to (4) and to the compactness of the Hardy-type operator (5) (cf. Corollary 3 below). (Note that if $r=+\infty$, then our conditions are sufficient; under some additional assumptions, they are also necessary-cf. Remarks 6 and 7 below.)

Finally, we apply our results to the case when $X\left(\mathbb{R}^{n}\right)$ is the Lorentz-Karamata space $L_{p, q ; b}\left(\mathbb{R}^{n}\right)$. The corresponding continuous embeddings are characterized in Theorems 3 and 4 below. The former concerns the superlimiting case when $p>n / \sigma$ while the latter is devoted to the limiting case when $p=n / \sigma$. In particular, we are able to characterize optimal embeddings of Bessel potential spaces $H^{\sigma} L_{p, q ; b}\left(\mathbb{R}^{n}\right)$ into generalized Hölder spaces and the continuity envelopes of Bessel potential spaces $H^{\sigma} L_{p, q ; b}\left(\mathbb{R}^{n}\right)$ both in superlimiting and limiting cases (cf. Remarks 4 and 5 below). The compact embeddings of Bessel potential spaces modelled upon Lorentz-Karamata spaces $L_{p, q ; b}\left(\mathbb{R}^{n}\right)$ into generalized Hölder spaces are characterized in Theorem 7 in the superlimiting case and in Theorem 8 in the limiting case. We refer to Remark 8 for the case $r=+\infty$. Our results extend and improve those of $[15,16,23]$.

The paper is organized as follows. Section 2 contains notation, definitions and preliminary assertions. Section 3 involves auxiliary results. In Sect. 4 we present sharp estimates of the modulus of smoothness of convolutions of functions from the rearrangement invariant space $X=X\left(\mathbb{R}^{n}\right)$ with the Bessel kernel. Such estimates play a key role in what follows. A characterization of continuous embeddings is given in Sect. 5 while in Sect. 6 we apply this result to Bessel-potential spaces modelled upon Lorentz-Karamata spaces. Compact subsets of generalized Hölder spaces are characterized in Sect. 7 while necessary and sufficient conditions for compact embeddings of Bessel potential spaces modelled upon LorentzKaramata spaces into generalized Hölder-type spaces are established in Sect. 8.

\section{Notation and preliminaries}

As usual, $\mathbb{R}^{n}$ denotes the Euclidean $n$-dimensional space. Throughout the paper $\mu_{n}$ is the $n$-dimensional Lebesgue measure in $\mathbb{R}^{n}$ and $\Omega$ is a $\mu_{n}$-measurable subset of $\mathbb{R}^{n}$. We denote by $\chi_{\Omega}$ the characteristic function of $\Omega$ and put $|\Omega|_{n}=\mu_{n}(\Omega)$. The family of all extended scalar-valued (real or complex) $\mu_{n}$-measurable functions on $\Omega$ is denoted by $\mathcal{M}(\Omega)$ while $\mathcal{M}^{+}(\Omega)$ stands for the subset of $\mathcal{M}(\Omega)$ consisting of all functions which are non-negative a.e. on $\Omega$. When $\Omega$ is an interval $(a, b) \subseteq \mathbb{R}$, we denote these sets by $\mathcal{M}(a, b)$ and $\mathcal{M}^{+}(a, b)$, respectively. By $\mathcal{M}^{+}(a, b ; \downarrow)$ we mean the subsets of $\mathcal{M}^{+}(a, b)$ containing all non-increasing functions on the interval $(a, b)$. The symbol $\mathcal{W}(a, b)$ stands for the class of weight functions on $(a, b) \subseteq \mathbb{R}$ consisting of all measurable functions which are positive and finite a.e. on $(a, b)$. The non-increasing rearrangement of $f \in \mathcal{M}(\Omega)$ is the function $f^{*}$ defined by $f^{*}(t):=\inf \left\{\lambda \geq 0:|\{x \in \Omega:|f(x)|>\lambda\}|_{n} \leq t\right\}$ for all $t \geq 0$. By $f^{* *}$ we denote the maximal function of $f^{*}$ given by $f^{* *}(t):=t^{-1} \int_{0}^{t} f^{*}(\tau) d \tau, t>0$.

Given a rearrangement-invariant Banach function space (r. i. BFS) $X$, the associate space is denoted by $X^{\prime}$. For general facts about rearrangement-invariant Banach function spaces we refer to [5].

Let $X$ and $Y$ be two (quasi-)Banach spaces. We say that $X$ coincides with $Y$ (and write $X=Y$ ) if $X$ and $Y$ are equal in the algebraic and topological sense (their (quasi-)norms are equivalent). The symbol $X \hookrightarrow Y$ or $X \hookrightarrow \hookrightarrow Y$ means that $X \subset Y$ and the natural embedding of $X$ in $Y$ is continuous or compact, respectively.

By $c, C, c_{1}, C_{1}, c_{2}, C_{2}$, etc. we denote positive constants independent of appropriate quantities. For two non-negative expressions (i.e. functions or functionals) $\mathcal{A}, \mathcal{B}$, the symbol 
$\mathcal{A} \precsim \mathcal{B}$ (or $\mathcal{A} \succsim \mathcal{B}$ ) means that $\mathcal{A} \leq c \mathcal{B}$ (or $c \mathcal{A} \geq \mathcal{B}$ ). If $\mathcal{A} \precsim \mathcal{B}$ and $\mathcal{A} \succsim \mathcal{B}$, we write $\mathcal{A} \approx \mathcal{B}$ and say that $\mathcal{A}$ and $\mathcal{B}$ are equivalent. Throughout the paper we use the abbreviation $\operatorname{LHS}(*)(\operatorname{RHS}(*))$ for the left- (right-) hand side of the relation $(*)$. We adopt the convention that $a /+\infty=0$ and $a / 0=+\infty$ for all $a>0$. If $p \in(0,+\infty]$, the conjugate number $p^{\prime}$ is given by $1 / p+1 / p^{\prime}=1$. Note that $p^{\prime}$ is negative, if $p \in(0,1)$. In the whole the paper $\|\cdot\|_{p ;(c, d)}, p \in(0,+\infty]$, stands for the usual $L^{p}$-(quasi-)norm on the interval $(c, d) \subseteq \mathbb{R}$.

For $\rho \in(0,+\infty)$ and $x \in \mathbb{R}^{n}, B(x, \rho)=B_{n}(x, \rho)$ stands for the open ball in $\mathbb{R}^{n}$ of radius $\rho$ and centre $x$. By $\beta_{n}$ we denote the volume of the unit ball in $\mathbb{R}^{n}$.

Following [24], we say that a positive and Lebesgue-measurable function $b$ is slowly varying on $(0,+\infty)$, and write $b \in S V(0,+\infty)$, if, for each $\epsilon>0, t^{\epsilon} b(t)$ is equivalent to a non-decreasing function on $(0,+\infty)$ and $t^{-\epsilon} b(t)$ is equivalent to a non-increasing function on $(0,+\infty)$. The family of all slowly varying functions includes not only powers of iterated logarithms and the broken logarithmic functions of [20], but also such functions as $t \rightarrow \exp \left(|\log t|^{a}\right), a \in(0,1)$. (The last mentioned function has the interesting property that it tends to infinity more quickly than any positive power of the logarithmic function.)

We shall need the following properties of slowly varying functions. We refer to [24, Proposition 2.2] for properties (i)-(iii); property (iv) is a simple consequence of the definition.

Lemma 1 Let $b, b_{1}$ and $b_{2}$ belong to $S V(0,+\infty)$. Then

(i) $b_{1} b_{2} \in S V(0,+\infty)$ and $b^{r} \in S V(0,+\infty)$ for each $r \in \mathbb{R}$;

(ii) given positive numbers $\varepsilon$ and $\kappa$, there are positive constants $c_{\varepsilon}$ and $C_{\varepsilon}$ such that

$$
c_{\varepsilon} \min \left\{\kappa^{-\varepsilon}, \kappa^{\varepsilon}\right\} b(t) \leq b(\kappa t) \leq C_{\varepsilon} \max \left\{\kappa^{-\varepsilon}, \kappa^{\varepsilon}\right\} b(t) \quad \text { for all } t>0 ;
$$

(iii) if $\alpha>0$ and $q \in(0, \infty]$, then for all $t>0$,

$$
\left\|\tau^{\alpha-1 / q} b(\tau)\right\|_{q,(0, t)} \approx t^{\alpha} b(t) \quad \text { and } \quad\left\|\tau^{-\alpha-1 / q} b(\tau)\right\|_{q,(t, \infty)} \approx t^{-\alpha} b(t)
$$

(iv) if $\alpha>0$, then

$$
t^{\alpha} b(t) \rightarrow 0 \text { as } t \rightarrow 0_{+} .
$$

We can see from Lemma 1 (iii) that any $b \in S V(0,+\infty)$ is equivalent to some $\widetilde{b} \in$ $S V(0,+\infty)$ which is continuous on $(0,+\infty)$. Consequently, without loss of generality, we shall assume that all slowly varying functions in question are continuous on $(0,+\infty)$.

More properties and examples of slowly varying functions can be found in $[6,17,24,27,32]$ and [40, Chapter V, p. 186].

We shall need the following weighted Hardy inequalities, for which we refer to [34, Theorems 5.9, 5.10 and 9.3]. The case $r \in(0,1)$ and $q=+\infty$ can be proved as the case $1 \leq r<+\infty$ and $q=+\infty$ in [28, Theorem 1.3.1/2].

Lemma 2 Let $q \in[1,+\infty], r \in(0,+\infty], a \in(0,+\infty]$ and $v, w \in \mathcal{W}(0, a)$.

(i) If $1 \leq q \leq r \leq+\infty$, then

$$
\left\|w(t) \int_{0}^{t} h(s) d s\right\|_{r ;(0, a)} \leq C\|v(t) h(t)\|_{q ;(0, a)} \text { for all } h \in \mathcal{M}^{+}(0, a)
$$

if and only if

$$
A:=\sup _{x \in(0, a)}\|w(t)\|_{r ;(x, a)}\left\|(v(t))^{-1}\right\|_{q^{\prime} ;(0, x)}<+\infty .
$$


Moreover, the best possible constant $C$ in (7) satisfies the estimate $C \approx A$ and the constants involved in this equivalence are independent of $a$.

(ii) If $0<r<q \leq+\infty$ and $q>1$, then (7) holds if and only if

$$
B:=\left(\int_{0}^{a}\left[\|w(t)\|_{r ;(x, a)}\left\|(v(t))^{-1}\right\|_{q^{\prime} ;(0, x)}^{q^{\prime} / r^{\prime}}\right]^{u} v^{-q^{\prime}}(x) d x\right)^{1 / u}<+\infty,
$$

where $\frac{1}{u}=\frac{1}{r}-\frac{1}{q}$. Moreover, the best possible constant $C$ in (7) satisfies $C \approx B$ and the constants involved in this equivalence are independent of $a$.

We shall also need the following weighted Hardy inequality, where weights involve slowly varying functions. Such an inequality is a consequence of [34, Theorem 6.2.].

Lemma 3 Let $1 \leq q \leq r \leq+\infty, a \in(0,+\infty], v \in \mathbb{R} \backslash\{0\}$ and let $b \in S V(0,+\infty)$. Then

$$
\left\|t^{\nu-1 / r} b(t) \int_{t}^{a} g(s) d s\right\|_{r ;(0, a)} \leq C\left\|t^{\nu+1 / q^{\prime}} b(t) g(t)\right\|_{q ;(0, a)} \text { for all } g \in \mathcal{M}^{+}(0, a)
$$

if and only if $v>0$. The positive constant $C$ in (10) can be chosen independent of $a$.

Let $p, q \in(0,+\infty], b \in S V(0,+\infty)$ and let $\Omega$ be a measurable subset of $\mathbb{R}^{n}$. The Lorentz-Karamata $(\mathrm{LK})$ space $L_{p, q ; b}(\Omega)$ is defined to be the set of all functions $f \in \mathcal{M}(\Omega)$ such that

$$
\|f\|_{p, q ; b ; \Omega}:=\left\|t^{1 / p-1 / q} b(t) f^{*}(t)\right\|_{q ;(0,+\infty)}<+\infty .
$$

If $\Omega=\mathbb{R}^{n}$, we simply write $\|\cdot\|_{p, q ; b}$ instead of $\|\cdot\|_{p, q ; b ; \mathbb{R}^{n}}$.

When $0<p<+\infty$, the Lorentz-Karamata space $L_{p, q ; b}(\Omega)$ contains the characteristic function of every measurable subset of $\Omega$ with finite measure and hence, by linearity, every $\mu_{n}$-simple function. When $p=+\infty$, the Lorentz-Karamata space $L_{p, q ; b}(\Omega)$ is different from the trivial space if and only if

$$
\left\|t^{1 / p-1 / q} b(t)\right\|_{q ;(0,1)}<+\infty .
$$

Particular choices of $b$ give well-known spaces. If $m \in \mathbb{N}, \boldsymbol{\alpha}=\left(\alpha_{1}, \ldots, \alpha_{m}\right) \in \mathbb{R}^{m}$ and

$$
b(t)=\ell^{\alpha}(t):=\prod_{i=1}^{m} l_{i}^{\alpha_{i}}(t) \text { for all } t>0
$$

(where $l_{1}(t)=1+|\log t|, l_{i}(t)=l_{1}\left(l_{i-1}(t)\right)$ if $i>1$ ), then the LK-space $L_{p, q ; b}(\Omega)$ is the generalized Lorentz-Zygmund space $L_{p, q, \boldsymbol{\alpha}}$ introduced in [13] and endowed with the (quasi-)norm $\|f\|_{p, q ; \boldsymbol{\alpha} ; \Omega}$, which in turn becomes the Lorentz-Zygmund space $L^{p, q}(\log L)^{\alpha_{1}}$ of Bennett and Rudnick [4] when $m=1$. If $\alpha=(0, \ldots, 0)$, we obtain the Lorentz space $L^{p, q}(\Omega)$ endowed with the (quasi-)norm $\|\cdot\|_{p, q ; \Omega}$, which is just the Lebesgue space $L^{p}(\Omega)$ equipped with the (quasi-) norm $\|\cdot\|_{p ; \Omega}$ when $p=q$; if $p=q$ and $m=1$, we obtain the Zygmund space $L^{p}(\log L)^{\alpha_{1}}(\Omega)$ endowed with the (quasi-)norm $\|.\|_{p ; \alpha_{1} ; \Omega}$.

The Riesz kernel $I_{\sigma}, 0<\sigma<n$, is defined by

$$
I_{\sigma}(x)=|x|^{\sigma-n}, \quad x \in \mathbb{R}^{n} .
$$

It is easy to show that

$$
\left(I_{\sigma}\right)^{*}(t)=\left(\frac{t}{\beta_{n}}\right)^{\frac{\sigma-n}{n}}, \quad t>0 .
$$


The Bessel kernel $g_{\sigma}, \sigma>0$, is defined as that function on $\mathbb{R}^{n}$ whose Fourier transform is $\widehat{g_{\sigma}}(\xi)=(2 \pi)^{-n / 2}\left(1+|\xi|^{2}\right)^{-\sigma / 2}, \quad \xi \in \mathbb{R}^{n}$, where the Fourier transform $\hat{f}$ of a function $f$ is given by $\hat{f}(\xi)=(2 \pi)^{-n / 2} \int_{\mathbb{R}^{n}} e^{-i \xi \cdot x} f(x) d x$.

Let us summarize the basic properties of the Bessel kernel $g_{\sigma}$ :

$g_{\sigma}$ is a positive, integrable function which is analytic except at the origin;

$g_{\sigma}$ is radially decreasing;

$$
\begin{aligned}
g_{\sigma}(x) \leq c_{1}|x|^{\sigma-n} e^{-c_{2}|x|} & \text { for } \quad 0<\sigma<n \quad \text { and all } \quad x \in \mathbb{R}^{n} \backslash\{0\} ; \\
g_{\sigma}(x) \approx|x|^{\sigma-n} & \text { as } \quad|x| \rightarrow 0 \quad \text { if } \quad 0<\sigma<n ;
\end{aligned}
$$

$$
\begin{gathered}
\left|\frac{\partial}{\partial x_{j}} g_{\sigma}(x)\right| \leq c|x|^{\sigma-n-1} \text { for } 0<\sigma \leq n+1, j \in\{1, \ldots, n\} \quad \text { and all } x \in \mathbb{R}^{n} \backslash\{0\} ; \\
g_{\sigma}^{*}(t) \precsim t^{(\sigma-n) / n} e^{-c t^{1 / n}} \text { for } 0<\sigma<n \quad \text { and all } t>0 .
\end{gathered}
$$

Property (13) follows from equation (26) in [36, Chap. V]. For the proof of (12), (14)-(16) see [3], for (17) see [12].

Let $\sigma>0$ and let $X=X\left(\mathbb{R}^{n}\right)=X\left(\mathbb{R}^{n}, \mu_{n}\right)$ be a r. i. Banach function space endowed with the norm $\|\cdot\|_{X}$. The Bessel potential space $H^{\sigma} X\left(\mathbb{R}^{n}\right)$ is defined by

$$
H^{\sigma} X\left(\mathbb{R}^{n}\right):=\left\{u: u=f * g_{\sigma}, f \in X\left(\mathbb{R}^{n}\right)\right\}
$$

and is equipped with the norm

$$
\|u\|_{H^{\sigma} X}:=\|f\|_{X} .
$$

Note that, given $f \in X$, the convolution $u=f * g_{\sigma}$ is well defined and finite $\mu_{n^{-}}$ a.e. on $\mathbb{R}^{n}$ since the measure space $\left(\mathbb{R}^{n}, \mu_{n}\right)$ is resonant and so (cf. [5, Theorem II.6.6]) $X \hookrightarrow L^{1}\left(\mathbb{R}^{n}\right)+L^{\infty}\left(\mathbb{R}^{n}\right)$.

If $p \in(1,+\infty], q \in[1,+\infty]$ and $b \in S V(0,+\infty)$, then the space $L_{p, q ; b}\left(\mathbb{R}^{n}\right)$ coincides with a r. i. Banach function space $X\left(\mathbb{R}^{n}\right)$ (the (quasi-)norm (11) is equivalent to the norm $\left\|t^{1 / p-1 / q} b(t) f^{* *}(t)\right\|_{q ;(0,+\infty)}$, which follows from the estimate $f^{*} \leq f^{* *}$ and Lemma 2(i) with $r=q, w(t)=t^{1 / p-1 / q-1} b(t), v(t)=t^{1 / p-1 / q} b(t)$ and $\left.a=+\infty\right)$. Consequently, if $\sigma>0, p \in(1,+\infty], q \in[1,+\infty]$ and $b \in S V(0,+\infty), H^{\sigma} L_{p, q ; b}\left(\mathbb{R}^{n}\right):=H^{\sigma} X\left(\mathbb{R}^{n}\right)$ is the usual Bessel potential space modelled upon the Lorentz-Karamata space $L_{p, q ; b}\left(\mathbb{R}^{n}\right)$, which is equipped with the (quasi-)norm

$$
\|u\|_{\sigma ; p, q ; b}:=\|f\|_{p, q ; b} .
$$

When $m \in \mathbb{N}, \boldsymbol{\alpha}=\left(\alpha_{1}, \ldots, \alpha_{m}\right) \in \mathbb{R}^{m}$ and $b=\ell^{\boldsymbol{\alpha}}$, we obtain the logarithmic Bessel potential space $H^{\sigma} L_{p, q ; \boldsymbol{\alpha}}\left(\mathbb{R}^{n}\right)$, endowed with the (quasi-)norm $\|u\|_{\sigma ; p, q ; b}$ and considered in [13]. Note that if $\boldsymbol{\alpha}=(0, \ldots, 0), H^{\sigma} L_{p, p ; \boldsymbol{\alpha}}\left(\mathbb{R}^{n}\right)$ is simply the (fractional) Sobolev space $H^{\sigma, p}\left(\mathbb{R}^{n}\right)$ of order $\sigma$.

When $k \in \mathbb{N}, p, q \in(1,+\infty)$ and $b \in S V(0,+\infty)$, then

$$
H^{k} L_{p, q ; b}\left(\mathbb{R}^{n}\right)=\left\{u: D^{\alpha} u \in L_{p, q ; b}\left(\mathbb{R}^{n}\right) \text { if }|\alpha| \leq k\right\},
$$

and

$$
\|u\|_{k ; p, q ; b} \approx \sum_{|\alpha| \leq k}\left\|D^{\alpha} u\right\|_{p, q ; b} \text { for all } u \in H^{k} L_{p, q ; b}\left(\mathbb{R}^{n}\right)
$$

according to [22, Lemma 4.5] and [33, Theorem 5.3]. 
Let $\Omega$ be a domain in $\mathbb{R}^{n}$. The space of all scalar-valued (real or complex), bounded and continuous functions on $\Omega$ is denoted by $C_{B}(\Omega)$ and it is equipped with the $L^{\infty}(\Omega)$-norm. For each $h \in \mathbb{R}^{n}$, let $\Omega_{h}=\{x \in \Omega: x+h \in \Omega\}$ and let $\Delta_{h}$ be the difference operator given on scalar functions $f$ on $\Omega$ by $\left(\Delta_{h} f\right)(x)=f(x+h)-f(x)$ for all $x \in \Omega_{h}$. The modulus of smoothness of a function $f$ in $C_{B}(\Omega)$ is defined by

$$
\omega(f, t):=\sup _{|h| \leq t}\left\|\Delta_{h} f \mid L^{\infty}\left(\Omega_{h}\right)\right\| \text { for all } t \geq 0 .
$$

If

$$
\widetilde{\omega}(f, t):=\omega(f, t) / t \text { for all } t>0,
$$

then $\widetilde{\omega}(f,$.$) is equivalent to a non-increasing function on (0,+\infty)$. The function $f \in C_{B}(\Omega)$ is uniformly continuous on $\Omega$ if and only if $\omega(f, t) \rightarrow \omega(f, 0)=0$ as $t \rightarrow 0_{+}$. We refer to [5, pp. 331-333] and to [9, pp. 40-50] for more details.

Let $\Omega$ be a domain in $\mathbb{R}^{n}$. By $C(\bar{\Omega})$ we mean the subspace of $C_{B}(\Omega)$ of all bounded and uniformly continuous functions on $\Omega$. A subset $S$ of $C(\bar{\Omega})$ is equicontinuous if and only if

$$
\sup _{f \in S} \omega(f, t) \rightarrow 0 \text { as } t \rightarrow 0_{+} .
$$

Let $r \in(0,+\infty]$ and let $\mathcal{L}_{r}$ be the class of all continuous functions $\lambda:(0,1] \rightarrow(0,+\infty)$ which are increasing on some interval $(0, \delta)$, with $\delta=\delta_{\lambda} \in(0,1]$, and satisfy

$$
\lim _{t \rightarrow 0_{+}} \lambda(t)=0
$$

and

$$
\left\|t^{-1 / r} \frac{t}{\lambda(t)}\right\|_{r ;(0, \delta)}<+\infty .
$$

When $r=+\infty$, we simply write $\mathcal{L}$ instead of $\mathcal{L}_{r}$.

If $\lambda \in \mathcal{L}_{r}$, one can easy see that $\lambda$ is equivalent to a continuous increasing function on the interval $(0,1]$. Consequently, without loss of generality, we shall assume that all elements of $\mathcal{L}_{r}$ are continuous increasing functions on the interval $(0,1]$.

Let $r \in(0,+\infty], \lambda \in \mathcal{L}_{r}$ and let $\Omega$ be a domain in $\mathbb{R}^{n}$. The generalized Hölder space $\Lambda_{\infty, r}^{\lambda(\cdot)}(\bar{\Omega})$ consists of all those functions $f \in C_{B}(\Omega)$ for which the quasi-norm

$$
\left\|f\left|\Lambda_{\infty, r}^{\lambda(\cdot)}(\bar{\Omega})\|:=\| f\right| L^{\infty}(\Omega)\right\|+\left\|t^{-1 / r} \frac{\omega(f, t)}{\lambda(t)}\right\|_{r ;(0,1)}
$$

is finite. Standard arguments show that the space $\Lambda_{\infty, r}^{\lambda(\cdot)}(\bar{\Omega})$ is complete (cf. [30, Theorem 3.1.4]). If (21) does not hold, then the space $\Lambda_{\infty, r}^{\lambda(\cdot)}(\bar{\Omega})$ contains only constant functions.

The space $\Lambda_{\infty, \infty}^{\lambda(.)}(\bar{\Omega})$ coincides (cf. [31, Proposition 3.5]) with the space $C^{0, \lambda(.)}(\bar{\Omega})$ defined by

$$
\left\|f\left|C^{0, \lambda(\cdot)}(\bar{\Omega}) \|:=\sup _{x \in \Omega}\right| f(x) \mid+\sup _{\substack{x, y \in \Omega \\ 0<|x-y| \leq 1}} \frac{|f(x)-f(y)|}{\lambda(|x-y|)}<+\infty .\right.
$$

If $\lambda(t)=t, t \in(0,1]$, and $\Omega=\mathbb{R}^{n}$, then $\Lambda_{\infty, \infty}^{\lambda(\cdot)}(\bar{\Omega})$ coincides with the space Lip $\left(\mathbb{R}^{n}\right)$ of the Lipschitz functions. If $\lambda(t) \equiv t^{\alpha}, \alpha \in(0,1]$, then the space $\Lambda_{\infty, r}^{\lambda(\cdot)}(\bar{\Omega})$ coincides with the space $C^{0, \alpha, r}(\bar{\Omega})$ introduced in [1]. 
The next lemma shows that we could define the generalized Hölder space $\Lambda_{\infty, r}^{\lambda(\cdot)}(\bar{\Omega})$ as a subspace of $C(\bar{\Omega})$ rather than a subspace of $C_{B}(\Omega)$.

Lemma 4 Let $r \in(0,+\infty], \lambda \in \mathcal{L}_{r}$ and let $\Omega$ be a domain in $\mathbb{R}^{n}$. Then

$$
\Lambda_{\infty, r}^{\lambda(\cdot)}(\bar{\Omega}) \hookrightarrow C(\bar{\Omega})
$$

Proof Let $f \in \Lambda_{\infty, r}^{\lambda(\cdot)}(\bar{\Omega})$. Then there is $M \in(0,+\infty)$ such that

$$
\left\|t^{-1 / r} \frac{\omega(f, t)}{\lambda(t)}\right\|_{r ;(0,1)}<M
$$

Since also, for all $t \in(0,1)$,

$$
\left\|\tau^{-1 / r} \frac{\omega(f, \tau)}{\lambda(\tau)}\right\|_{r ;(0, t)}=\left\|\tau^{1-1 / r} \frac{\widetilde{\omega}(f, \tau)}{\lambda(\tau)}\right\|_{r ;(0, t)} \succsim \frac{\widetilde{\omega}(f, t)}{\lambda(t)}\left\|\tau^{1-1 / r}\right\|_{r ;(0, t)} \approx \frac{\omega(f, t)}{\lambda(t)},
$$

we obtain,

$$
\omega(f, t) \precsim \lambda(t) \text { for all } t \in(0,1) .
$$

Moreover, the fact that $\lambda(t) \rightarrow 0$ as $t \rightarrow 0_{+}$yields $\omega(f, t) \rightarrow 0$ as $t \rightarrow 0_{+}$. Consequently, $f$ is uniformly continuous on $\Omega$.

Let $\Omega$ be a measurable subset of $\mathbb{R}^{n}$. We denote by $B(\Omega)$ the set of all scalar-valued functions (real or complex) which are bounded on $\Omega$ and we equip this set with the norm

$$
\|f\|_{B(\Omega)}:=\sup \{|f(x)|: x \in \Omega\} .
$$

In the paper we investigate embeddings of the form

$$
H^{\sigma} X\left(\mathbb{R}^{n}\right) \hookrightarrow Y(\Omega),
$$

where $\sigma>0, X=X\left(\mathbb{R}^{n}\right)$ is a r. i. Banach function space, $\Omega$ is a domain in $\mathbb{R}^{n}$ and $Y(\Omega)$ is a convenient Banach space of functions defined on $\Omega$. Note that embedding (22) means that the mapping $\left.u \mapsto u\right|_{\Omega}$ from $H^{\sigma} X\left(\mathbb{R}^{n}\right)$ into $Y(\Omega)$ is continuous. Note also that in the whole paper we use the symbol $u$ both for the function $u$ and its restriction to $\Omega$.

\section{Auxiliary results}

First, we need the following assertion.

Lemma 5 Let $X=X\left(\mathbb{R}^{n}\right)$ be a r. i. BFS. Suppose that $f \in X$ and put $\bar{f}(x):=f^{*}\left(\beta_{n} \frac{|x|^{n}}{2^{n}}\right)$, $x \in \mathbb{R}^{n}$. Then $\bar{f} \in X$.

Proof Let $\bar{X}=\bar{X}\left((0,+\infty), \mu_{1}\right)$ be the representation space of $X$ (cf. [5, Chap. 2, Theorem 4.10]; by [5, p. 147], $\bar{X}$ is given uniquely). Let $f \in X$. Then, by Proposition 5.11 of [5, Chap. 3], the function $h(t):=f^{*}\left(\frac{t}{2^{n}}\right), t \in(0,+\infty)$, satisfies $h \in \bar{X}$. One can also easily verify that $(\bar{f})^{*}=h$. Consequently, $\|\bar{f}\|_{X}=\|h\|_{\bar{X}}<+\infty$ and the result follows.

Remark 1 There is another proof of Lemma 5. Indeed, using the hyperplanes $x_{i}=0$, $i=1, \ldots, n$, we decompose the space $\mathbb{R}^{n}$ into $2^{n}$ sets $Q_{k}, k=1, \ldots, 2^{n}$, with disjoint interiors such that

$$
\mathbb{R}^{n}=\bigcup_{k=1}^{2^{n}} Q_{k}
$$


Let $f_{k}(x)=f^{*}\left(\beta_{n} \frac{|x|^{n}}{2^{n}}\right) \chi_{Q_{k}}(x), x \in \mathbb{R}^{n}$. Then, $\left(f_{k}\right)^{*}(t)=f^{*}(t)$ for all $t>0$. Since $f \in X$, we have $f_{k} \in X$ for each $k=1, \ldots, 2^{n}$. Hence $\bar{f}=\sum_{k=1}^{2^{n}} f_{k} \in X$.

The next lemma is related to [13, Lemma 4.5].

Lemma 6 Let $X=X\left(\mathbb{R}^{n}\right)$ be a r. i. BFS and let $\Omega$ be a domain in $\mathbb{R}^{n}$. Suppose that $\sigma>0$ and let $g_{\sigma}$ be the Bessel kernel. Then

$$
H^{\sigma} X\left(\mathbb{R}^{n}\right) \hookrightarrow B(\Omega)
$$

if and only if

$$
\left\|g_{\sigma}\right\|_{X^{\prime}}<+\infty
$$

Proof Suppose that $\left\|g_{\sigma}\right\|_{X^{\prime}}<+\infty$. Let $u=f * g_{\sigma}$, where $f \in X$. By Hölder's inequality (cf. [5, Chap. 2, Corollary 4.5]),

$$
|u(x)| \leq \int_{\mathbb{R}^{n}}|f(y)| g_{\sigma}(x-y) d y \leq\|f\|_{X}\left\|g_{\sigma}\right\|_{X^{\prime}} \quad \text { for all } \quad x \in \Omega .
$$

Therefore,

$$
\|u\|_{B(\Omega)} \leq\left\|g_{\sigma}\right\|_{X^{\prime}}\|f\|_{X},
$$

which, together with (19), gives (23).

Suppose now that (23) holds. Take $f \in X$ and $x_{0} \in \Omega$. Put $\widetilde{f}(x)=\bar{f}\left(x-x_{0}\right), x \in \mathbb{R}^{n}$, where $\bar{f}(x)=f^{*}\left(\beta_{n} \frac{|x|^{n}}{2^{n}}\right), x \in \mathbb{R}^{n}$. Since $(\tilde{f})^{*}=(\bar{f})^{*}$ and $\bar{f} \in X$ by Lemma 5 , we have $\widetilde{f} \in X$. Hence, $u:=\widetilde{f} * g_{\sigma} \in H^{\sigma} X$. Moreover, since $\left|x-y-x_{0}\right| \leq\left|x-x_{0}\right|+|y| \leq 2|y|$ if $\left|x-x_{0}\right| \leq|y|$ and since $\bar{f}, g_{\sigma}$ are radially decreasing, we obtain

$$
\begin{aligned}
|u(x)| & =\int_{\mathbb{R}^{n}} \tilde{f}(x-y) g_{\sigma}(y) d y \\
& =\int_{\mathbb{R}^{n}} \bar{f}\left(x-y-x_{0}\right) g_{\sigma}(y) d y \\
& \geq \int_{|y| \geq\left|x-x_{0}\right|} \bar{f}(2 y) g_{\sigma}(y) d y \\
& =\int_{|y| \geq\left|x-x_{0}\right|} f^{*}\left(\beta_{n}|y|^{n}\right) g_{\sigma}^{*}\left(\beta_{n}|y|^{n}\right) d y \\
& =\int_{\left|x-x_{0}\right|\{|y|=\rho\}}^{+\infty} f^{*}\left(\beta_{n}|y|^{n}\right) g_{\sigma}^{*}\left(\beta_{n}|y|^{n}\right) d \vartheta d \rho \\
& =\int_{\left|x-x_{0}\right|}^{+\infty} f^{*}\left(\beta_{n} \rho^{n}\right) g_{\sigma}^{*}\left(\beta_{n} \rho^{n}\right) n \beta_{n} \rho^{n-1} d \rho \\
& =\int_{\beta_{n}\left|x-x_{0}\right|^{n}}^{+\infty} f^{*}(s) g_{\sigma}^{*}(s) d s, \quad x \in \Omega .
\end{aligned}
$$


Together with the fact $u \in B(\Omega)$ (cf. (23)), this yields

$$
+\infty>\|u\|_{B(\Omega)} \geq \int_{0}^{+\infty} f^{*}(s) g_{\sigma}^{*}(s) d s .
$$

Since inequality (26) holds for any $f \in X, g_{\sigma}$ belongs to $X^{\prime}$, which gives (24).

Remark 2 Let $\sigma \in(0,1)$ and let $X=X\left(\mathbb{R}^{n}\right)$ be a r. i. BFS such that $\left\|g_{\sigma}\right\|_{X^{\prime}}<+\infty$. Then

$$
\int_{0}^{1} s^{\frac{\sigma}{n}-1} f^{*}(s) d s<+\infty \text { for all } f \in X
$$

(which implies that a function $f \in X\left(\mathbb{R}^{n}\right)$ belongs to the Lorentz space $L^{\frac{n}{\sigma}, 1}(B)$ for any ball $\left.B \subset \mathbb{R}^{n}\right)$. Indeed, we deduce from (15) and (12) that $g_{\sigma}^{*}(s) \approx s^{\frac{\sigma}{n}-1}$ for all $s \in(0,1)$ and so the result follows from the Hölder inequality.

\section{Key estimates}

In the next theorem we present sharp estimates of modulus of smoothness of the convolution of a function $f$ from a r. i. BFS $X\left(\mathbb{R}^{n}\right)$ with the Bessel potential kernel $g_{\sigma}, 0<\sigma<1$. Such estimates are essential in what follows.

Theorem 1 Let $\sigma \in(0,1)$ and let $X=X\left(\mathbb{R}^{n}\right)$ be a r. i. BFS such that $\left\|g_{\sigma}\right\|_{X^{\prime}}<+\infty$. Then $f * g_{\sigma} \in C_{B}\left(\mathbb{R}^{n}\right)$ for all $f \in X$ and

$$
\omega\left(f * g_{\sigma}, t\right) \precsim \int_{0}^{t^{n}} s^{\frac{\sigma}{n}-1} f^{*}(s) d s \text { for all } t \in(0,1) \text { and every } f \in X .
$$

Moreover, estimate (28) is sharp in the sense that

$$
\omega\left(\bar{f} * g_{\sigma}, t\right) \succsim \int_{0}^{t^{n}} s^{\frac{\sigma}{n}-1} f^{*}(s) d s \text { for all } t \in(0,1) \text { and every } f \in X,
$$

where

$$
\bar{f}(x):=f^{*}\left(\beta_{n}|x|^{n}\right) \chi\left\{y \in \mathbb{R}^{n}: y_{1}>0\right\} \cap B(0,1)(x), \quad x=\left(x_{1}, \cdots, x_{n}\right) \in \mathbb{R}^{n} .
$$

(Note that $\bar{f}^{*}(t)=f^{*}(2 t)$ for all $\left.t \in\left(0, \beta_{n} / 2\right)\right)$.

Proof (i) Let $h \in \mathbb{R}^{n}, 0<|h|<1$, and let $x \in \mathbb{R}^{n}$. Put

$$
B_{1}:=B\left(x, \frac{|h|}{2}\right), \quad B_{2}:=B\left(x+h, \frac{|h|}{2}\right), \quad B_{3}:=B(x, 2|h|) \backslash\left(B_{1} \cup B_{2}\right)
$$

and

$$
B_{4}:=B^{c}(x, 2|h|):=\mathbb{R}^{n} \backslash B(x, 2|h|) .
$$


Take $f \in X\left(\mathbb{R}^{n}\right)$ and put $u=f * g_{\sigma}$. Then, by Lemma 6 with $\Omega=\mathbb{R}^{n}, u \in B\left(\mathbb{R}^{n}\right)$, which implies that $\left(\Delta_{h} u\right)(x)$ is defined for all $x \in \mathbb{R}^{n}$. Since

$$
\left(\Delta_{h} u\right)(x)=\int_{\mathbb{R}^{n}} f(y)\left(\Delta_{h} g_{\sigma}\right)(x-y) d y \text { for all } x \in \mathbb{R}^{n},
$$

we have

$$
\left|\left(\Delta_{h} u\right)(x)\right| \leq N_{1}+N_{2}+N_{3}+N_{4},
$$

where

$$
\begin{aligned}
& N_{1}:=\int_{B_{1}}|f(y)|\left|\left(\Delta_{h} g_{\sigma}\right)(x-y)\right| d y, \\
& N_{2}:=\int_{B_{2}}|f(y)|\left|\left(\Delta_{h} g_{\sigma}\right)(x-y)\right| d y, \\
& N_{3}:=\int_{B_{3}}|f(y)|\left|\left(\Delta_{h} g_{\sigma}\right)(x-y)\right| d y, \\
& N_{4}:=\int_{B_{4}}|f(y)|\left|\left(\Delta_{h} g_{\sigma}\right)(x-y)\right| d y .
\end{aligned}
$$

If $y \in B_{1}$, then $|h|>2|x-y|$ and

$$
|x+h-y| \geq|h|-|x-y|>|x-y| .
$$

This and (13) yield, for all $y \in B_{1} \backslash\{x\}$,

$$
\left|\left(\Delta_{h} g_{\sigma}\right)(x-y)\right| \leq\left|g_{\sigma}(x+h-y)\right|+\left|g_{\sigma}(x-y)\right| \precsim g_{\sigma}(x-y) .
$$

Consequently,

$$
N_{1} \precsim \int_{B_{1}}|f(y)| g_{\sigma}(x-y) d y .
$$

Hardy's lemma, cf. [5, Chapter 2, Theorem 2.2], and (17) give

$$
N_{1} \precsim \int_{0}^{\beta_{n}(|h| / 2)^{n}} f^{*}(s) s^{\frac{\sigma-n}{n}} d s \approx \int_{0}^{|h|^{n}} f^{*}(s) s^{\frac{\sigma-n}{n}} d s .
$$

Analogously, we arrive at

$$
N_{2} \precsim \int_{B_{2}}|f(y)| g_{\sigma}(x-y+h) d y
$$

and

$$
N_{2} \precsim \int_{0}^{|h|^{n}} f^{*}(s) s^{\frac{\sigma-n}{n}} d s
$$


If $y \in B_{3}$, then $2|h|>|x-y| \geq \frac{|h|}{2}$ and $|x+h-y| \geq \frac{|h|}{2}$. Hence, $|x+h-y| \leq$ $|h|+|x-y|<3|h|$. Thus, by (12) and (15),

$$
g_{\sigma}(x-y) \approx|x-y|^{\sigma-n} \approx|h|^{\sigma-n} \text { for all } y \in B_{3}
$$

and

$$
g_{\sigma}(x+h-y) \approx|x+h-y|^{\sigma-n} \approx|h|^{\sigma-n} \text { for all } y \in B_{3} .
$$

This implies that

$$
\left|\left(\Delta_{h} g_{\sigma}\right)(x-y)\right| \leq\left|g_{\sigma}(x+h-y)\right|+\left|g_{\sigma}(x-y)\right| \approx|h|^{\sigma-n} \text { for all } y \in B_{3}
$$

and so

$$
N_{3} \precsim|h|^{\sigma-n} \int_{B_{3}}|f(y)| d y .
$$

Applying Hardy's lemma, we arrive at

$$
N_{3} \precsim|h|^{\sigma-n} \int_{0}^{\beta_{n}(2|h|)^{n}} f^{*}(s) d s \approx|h|^{\sigma-n} \int_{0}^{|h|^{n}} f^{*}(s) d s \precsim \int_{0}^{|h|^{n}} f^{*}(s) s^{\frac{\sigma-n}{n}} d s .
$$

To derive an estimate in the exterior of the ball $B(x, 2|h|)$, we use some ideas from the proof of [15, Lemma 2.5, p. 247]. The inequality

$$
\left|\Delta_{h} g_{\sigma}(x-y)\right| \leq|h| \sum_{j=1}^{n} \int_{0}^{1}\left|\frac{\partial}{\partial x_{j}} g_{\sigma}(x-y+\tau h)\right| d \tau \text { for all } x, y \in \mathbb{R}^{n}, x \neq y,
$$

together with the obvious estimate

$$
\frac{1}{2}|x-y| \leq|x-y+\tau h| \leq \frac{3}{2}|x-y| \text { if } \tau \in[0,1] \quad \text { and } \quad y \in B_{4}=B^{c}(x, 2|h|)
$$

and (16), yields

$$
\left|\Delta_{h} g_{\sigma}(x-y) \chi_{B^{c}(x, 2|h|)}(y)\right| \precsim|h||x-y|^{\sigma-n-1} \chi_{B^{c}(x, 2|h|)}(y), \quad y \in \mathbb{R}^{n} \backslash\{x\} .
$$

Thus

$$
N_{4} \precsim|h| \int_{\mathbb{R}^{n}}|f(y)||x-y|^{\sigma-n-1} \chi_{B^{c}(x,|h|)}(y) d y .
$$

Putting

$$
F(y)=|x-y|^{\sigma-n-1} \chi_{B^{c}(x,|h|)}(y), \quad y \in \mathbb{R}^{n} \backslash\{x\},
$$

and taking into account that $\sigma-n-1<0$, we can easily see that

$$
F^{*}(t)=\left(|h|^{n}+\frac{t}{\beta_{n}}\right)^{(\sigma-n-1) / n} \text { for all } t>0 .
$$

This and Hardy's lemma imply that

$$
N_{4} \precsim|h| \int_{0}^{+\infty} f^{*}(s)\left(|h|^{n}+\frac{s}{\beta_{n}}\right)^{(\sigma-n-1) / n} d s .
$$


On the other hand, because $\sigma<1$, we have

$$
\begin{aligned}
& |h| \int_{0}^{+\infty} \frac{f^{*}(s)}{\left(|h|^{n}+\frac{s}{\beta_{n}}\right)^{(n-\sigma+1) / n}} d s \\
& =|h| \int_{0}^{|h|^{n}} \frac{f^{*}(s)}{\left(|h|^{n}+\frac{s}{\beta_{n}}\right)^{(n-\sigma+1) / n}} d s \\
& +|h| \int_{|h|^{n}}^{+\infty} \frac{f^{*}(s)}{\left(|h|^{n}+\frac{s}{\beta_{n}}\right)^{(n-\sigma+1) / n}} d s \\
& \precsim|h|^{\sigma-n} \int_{0}^{|h|^{n}} f^{*}(s) d s+|h| \int_{|h|^{n}}^{+\infty} f^{*}(s) s^{(\sigma-n-1) / n} d s \\
& \precsim \int_{0}^{|h|^{n}} f^{*}(s) s^{\frac{\sigma-n}{n}} d s+|h| f^{*}\left(|h|^{n}\right) \int_{|h|^{n}}^{+\infty} s^{(\sigma-n-1) / n} d s \\
& \approx \int_{0}^{|h|^{n}} f^{*}(s) s^{\frac{\sigma-n}{n}} d s+|h| f^{*}\left(|h|^{n}\right)\left(|h|^{n}\right)^{(\sigma-1) / n} \\
& \approx \int_{0}^{|h|^{n}} f^{*}(s) s^{\frac{\sigma-n}{n}} d s+f^{*}\left(|h|^{n}\right)\left(|h|^{n}\right)^{\sigma / n} \\
& \precsim \int_{0}^{|h|^{n}} f^{*}(s) s^{\frac{\sigma-n}{n}} d s+\int_{\frac{|h|^{n}}{2}}^{|h|^{n}} f^{*}(s) s^{\frac{\sigma-n}{n}} d s \\
& \approx \int_{0}^{|h|^{n}} f^{*}(s) s^{\frac{\sigma-n}{n}} d s
\end{aligned}
$$

The last two estimates show that

$$
N_{4} \precsim \int_{0}^{|h|^{n}} f^{*}(s) s^{\frac{\sigma-n}{n}} d s .
$$

Therefore, by (31), (33), (35), (37) and (40), we obtain

$$
\left|\left(\Delta_{h} u\right)(x)\right| \precsim \int_{0}^{|h|^{n}} f^{*}(s) s^{\frac{\sigma-n}{n}} d s, \quad h \in \mathbb{R}^{n}, \quad 0<|h|<1, \text { for all } x \in \mathbb{R}^{n} .
$$

Together with (27), this implies that $u$ is continuous on $\mathbb{R}^{n}$. Thus (cf. Lemma 6 with $\Omega=\mathbb{R}^{n}$ ), the function $u$ belongs to $C_{B}\left(\mathbb{R}^{n}\right)$ and (28) follows from (41). 
(ii) Now we are going to prove (29). Let $\bar{u}=\bar{f} * g_{\sigma}$, where $f \in X$. Take $t \in(0,1)$ and put $\bar{t}=(-t, 0, \ldots, 0) \in \mathbb{R}^{n}$. Then

$$
\omega(\bar{u}, t) \geq|\bar{u}(\bar{t})-\bar{u}(0)| .
$$

If $y \in B(0,1)$ and $y_{1}>0$, then

$$
\begin{aligned}
|\bar{t}-y| & =\left(\left(-t-y_{1}\right)^{2}+\left(-y_{2}\right)^{2}+\cdots+\left(-y_{n}\right)^{2}\right)^{1 / 2} \\
& \geq\left(\left(-y_{1}\right)^{2}+\left(-y_{2}\right)^{2}+\cdots+\left(-y_{n}\right)^{2}\right)^{1 / 2}=|-y|=|y| .
\end{aligned}
$$

Thus, by (13),

$$
0 \geq g_{\sigma}(\bar{t}-y)-g_{\sigma}(-y)=g_{\sigma}(\bar{t}-y)-g_{\sigma}(y)
$$

Consequently,

$$
\begin{aligned}
|\bar{u}(\bar{t})-\bar{u}(0)| & =\int_{\left\{y \in \mathbb{R}^{n}: y_{1}>0\right\} \cap B(0,1)} f^{*}\left(\beta_{n}|y|^{n}\right)\left(g_{\sigma}(y)-g_{\sigma}(\bar{t}-y)\right) d y \\
& \geq \int_{\left\{y \in \mathbb{R}^{n}: y_{1}>0\right\} \cap B(0, k t)} f^{*}\left(\beta_{n}|y|^{n}\right)\left(g_{\sigma}(y)-g_{\sigma}(\bar{t}-y)\right) d y
\end{aligned}
$$

for any $k \in(0,1)$. If $|y| \leq k t$, then

$$
|\bar{t}-y| \geq|\bar{t}|-|y|=t-|y| \geq t-k t=(1-k) t \geq \frac{1-k}{k}|y|,
$$

that is,

$$
|y| \bar{k} \leq|\bar{t}-y|
$$

where $\bar{k}:=(1-k) / k$. Observe that $\bar{k} \rightarrow+\infty$ as $k \rightarrow 0_{+}$.

By (12) and (15),

$$
c_{1}|x|^{\sigma-n} \leq g_{\sigma}(x) \leq c_{2}|x|^{\sigma-n} \quad \text { for all } x \in B(0,2) \backslash\{0\} .
$$

Together with (44), this gives

$$
g_{\sigma}(\bar{t}-y) \leq c_{2}|\bar{t}-y|^{\sigma-n} \leq c_{2} \frac{|y|^{\sigma-n}}{\bar{k}^{n-\sigma}} \leq \frac{c_{2}}{c_{1} \bar{k}^{n-\sigma}} g_{\sigma}(y) \text { for all } y \in B(0, k t) \backslash\{0\} .
$$

Choose $k \in(0,1)$ small so that $\frac{c_{2}}{c_{1} \bar{k}^{n-\sigma}} \leq \frac{1}{2}$. Then,

$$
g_{\sigma}(\bar{t}-y) \leq \frac{1}{2} g_{\sigma}(y) \text { for all } y \in B(0, k t) \backslash\{0\} .
$$


Now, (42), (43), (46) and (45) yield

$$
\begin{aligned}
\omega(\bar{u}, t) & \succsim \int_{\left\{y \in \mathbb{R}^{n}: y_{1}>0\right\} \cap B(0, k t)} f^{*}\left(\beta_{n}|y|^{n}\right) g_{\sigma}(y) d y \\
& \approx \int_{\left\{y \in \mathbb{R}^{n}: y_{1}>0\right\} \cap B(0, k t)} f^{*}\left(\beta_{n}|y|^{n}\right)|y|^{\sigma-n} d y \\
& \approx \int_{0}^{(k t)^{n}} f^{*}(s) s^{\frac{\sigma-n}{n}} d s \\
& \approx \int_{0}^{t^{n}} f^{*}(s) s^{\frac{\sigma-n}{n}} d s
\end{aligned}
$$

and the proof is complete.

The following corollary is a consequence of Theorem 1 and Lemma 6.

Corollary 1 Let $\sigma \in(0,1)$ and let $X=X\left(\mathbb{R}^{n}\right)$ be a r. i. BFS. Assume that $\Omega$ is a domain in $\mathbb{R}^{n}$. Then $H^{\sigma} X\left(\mathbb{R}^{n}\right) \hookrightarrow C(\bar{\Omega})$ if and only if $\left\|g_{\sigma}\right\|_{X^{\prime}}<+\infty$.

Remark 3 In Sect. 7 we shall investigate the compactness of the embedding

$$
H^{\sigma} X\left(\mathbb{R}^{n}\right) \hookrightarrow \Lambda_{\infty, r}^{\mu(\cdot)}(\bar{\Omega}),
$$

where $\Omega$ will be a bounded domain in $\mathbb{R}^{n}$. Note that, by (47), the restriction to $\Omega$ of a function $u \in H^{\sigma} X\left(\mathbb{R}^{n}\right)$ belongs to the space $\Lambda_{\infty, r}^{\mu(\cdot)}(\bar{\Omega})$. Note also that $u=f * g_{\sigma}$ for some $f \in X\left(\mathbb{R}^{n}\right)$. Under the assumptions of Theorem $1, u \in C_{B}\left(\mathbb{R}^{n}\right)$, which implies that $u \in C_{B}(\Omega)$. To calculate $\left\|u \mid \Lambda_{\infty, r}^{\mu(\cdot)}(\bar{\Omega})\right\|$, we need the modulus of continuity of the function $u$. Clearly, $\omega(u, t)=\omega_{\Omega}(u, t), t \geq 0$, that is, the modulus of continuity depends on a given domain $\Omega$. Recall also that the modulus of continuity $\omega\left(f * g_{\sigma}, \cdot\right)$ involved in Theorem 1 is the modulus of continuity with respect to the whole $\mathbb{R}^{n}$, that is, $\omega_{\mathbb{R}^{n}}\left(f * g_{\sigma}, \cdot\right)$.

To characterize the compactness of the embedding (47), we shall need analogues of estimates (28) and (29) with $\omega$ replaced by $\omega_{\Omega}$. Since

$$
\omega_{\Omega}\left(f * g_{\sigma}, t\right) \leq \omega_{\mathbb{R}^{n}}\left(f * g_{\sigma}, t\right), \quad t \geq 0,
$$

estimate (28) implies that

$$
\omega_{\Omega}\left(f * g_{\sigma}, t\right) \precsim \int_{0}^{t^{n}} s^{\frac{\sigma}{n}-1} f^{*}(s) d s \text { for all } t \in(0,1) \text { and every } f \in X .
$$

To get an analogue of (29), take $x_{0}=\left(x_{01}, \ldots, x_{0 n}\right) \in \Omega$ and $r \in(0,1]$ so that $B\left(x_{0}, r\right) \subset \Omega$. Then

$$
\omega_{\Omega}\left(\bar{f} * g_{\sigma}, t\right) \succsim \int_{0}^{t^{n}} s^{\frac{\sigma}{n}-1} f^{*}(s) d s \text { for all } t \in(0,1) \text { and every } f \in X,
$$

where

$$
\bar{f}(x):=f^{*}\left(\beta_{n}\left|x-x_{0}\right|^{n}\right) \chi_{\left\{y \in \mathbb{R}^{n}: y_{1}>x_{01}\right\} \cap B\left(x_{0}, r\right)}(x), \quad x=\left(x_{1}, \ldots, x_{n}\right) \in \mathbb{R}^{n} .
$$



have

Indeed, take $t \in(0,1)$ and put $\bar{t}=(-t r, 0, \ldots, 0) \in \mathbb{R}^{n}$. Then, instead of (42), we now

$$
\omega_{\Omega}(\bar{u}, t) \geq\left|\bar{u}\left(\bar{t}+x_{0}\right)-\bar{u}\left(x_{0}\right)\right|
$$

and the same arguments as those used in part (ii) of the proof of Theorem 1 yield (49).

In what follows we shall omit the subscript $\Omega$ at the modulus of continuity since it will be always clear from the context which modulus of continuity we have in mind.

\section{A characterization of the continuous embedding}

Now, we are able to characterize the continuous embedding of the space $H^{\sigma} X\left(\mathbb{R}^{n}\right)$, with $\sigma \in(0,1)$ and a r. i. BFS $X=X\left(\mathbb{R}^{n}\right)$, into the generalized Hölder space $\Lambda_{\infty, r}^{\mu(\cdot)}\left(\overline{\mathbb{R}^{n}}\right)$. Assuming that $g_{\sigma} \in X^{\prime}$ in the next theorem, we reduce this problem to inequality (53) below, which involves the Hardy-type operator (6).

Theorem 2 Let $\sigma \in(0,1)$ and let $X=X\left(\mathbb{R}^{n}\right)=X\left(\mathbb{R}^{n}, \mu_{n}\right)$ be a r. i. BFS such that $\left\|g_{\sigma}\right\|_{X^{\prime}}<+\infty$. Assume that $r \in(0,+\infty]$ and $\mu \in \mathcal{L}_{r}$. Then

$$
H^{\sigma} X\left(\mathbb{R}^{n}\right) \hookrightarrow \Lambda_{\infty, r}^{\mu(\cdot)}\left(\overline{\mathbb{R}^{n}}\right)
$$

if and only if

$$
\left\|t^{-\frac{1}{r}}(\mu(t))^{-1} \int_{0}^{t^{n}} \tau^{\frac{\sigma}{n}-1} f^{*}(\tau) d \tau\right\|_{r ;(0,1)} \precsim\|f\|_{X} \text { for all } f \in X .
$$

Proof SufFICIENCY. Assume that (53) holds. Let $f \in X$ and $u=f * g_{\sigma}$. Then, by Theorem 1 ,

$$
\omega(u, t) \precsim \int_{0}^{t^{n}} s^{\frac{\sigma}{n}-1} f^{*}(s) d s \text { for all } t \in(0,1),
$$

which implies that

$$
\left\|t^{-\frac{1}{r}}(\mu(t))^{-1} \omega(u, t)\right\|_{r ;(0,1)} \precsim\left\|t^{-\frac{1}{r}}(\mu(t))^{-1} \int_{0}^{t^{n}} s^{\frac{\sigma}{n}-1} f^{*}(s) d s\right\|_{r ;(0,1)} .
$$

This, together with (53), yields

$$
\left\|t^{-\frac{1}{r}}(\mu(t))^{-1} \omega(u, t)\right\|_{r ;(0,1)} \precsim\|f\|_{X}=\|u\|_{H^{\sigma} X} .
$$

Thus, embedding (52) is a consequence of the last estimate and Corollary 1 (with $\Omega=\mathbb{R}^{n}$ ). NECESSITY. Suppose that (52) holds. Then, for all $h \in X$ and $u=h * g_{\sigma}$,

$$
\left\|t^{-\frac{1}{r}}(\mu(t))^{-1} \omega(u, t)\right\|_{r ;(0,1)} \precsim\|u\|_{H^{\sigma} X}=\|h\|_{X} .
$$

Let $f \in X$. Since $X$ is a r. i. Banach function space, the function $\bar{f}$ given by (30) also belongs to $X$ and $(\bar{f})^{*} \leq f^{*}$, which implies that

$$
\|\bar{f}\|_{X} \leq\|f\|_{X} .
$$


Putting $\bar{u}=\bar{f} * g_{\sigma}$, we have from Theorem 1 that

$$
\omega(\bar{u}, t) \succsim \int_{0}^{t^{n}} s^{\frac{\sigma}{n}-1} f^{*}(s) d s \text { for all } t \in(0,1) .
$$

Together with (54) and (55), this yields

$$
\left\|t^{-\frac{1}{r}}(\mu(t))^{-1} \int_{0}^{t^{n}} s^{\frac{\sigma}{n}-1} f^{*}(s) d s\right\|_{r ;(0,1)} \precsim\|\bar{f}\|_{X} \leq\|f\|_{X}
$$

and (53) is verified.

Using Theorem 2 and Lemma 6 with $\Omega=\mathbb{R}^{n}$, we arrive at the following corollary.

Corollary 2 Let $\sigma \in(0,1)$ and let $X=X\left(\mathbb{R}^{n}\right)$ be a r. i. BFS. Assume that $r \in(0,+\infty]$ and $\mu \in \mathcal{L}_{r}$. Then embedding (52) holds if and only if $\left\|g_{\sigma}\right\|_{X^{\prime}}<+\infty$ and (53) is satisfied.

\section{Applications of the embedding result}

We are interested in embeddings in the case when the space $X$ is a Lorentz-Karamata space $L_{p, q ; b}\left(\mathbb{R}^{n}\right)$ with $p \in(1,+\infty), q \in[1,+\infty]$ and $b \in S V(0,+\infty)$. Then $X$ coincides with a r. i. Banach function space and

$$
X^{\prime}=L_{p^{\prime}, q^{\prime} ; 1 / b}\left(\mathbb{R}^{n}\right)
$$

(see [32, Theorem 3.1] and replace $\gamma_{b}$ by $b$ and $\gamma_{1 / b}$ by $1 / b$ there).

To verify the assumption $\left\|g_{\sigma}\right\|_{X^{\prime}}<+\infty$ in Theorem 2 , we shall use the next lemma.

Lemma 7 Let $\sigma \in(0, n), p \in(1,+\infty), q \in[1,+\infty]$ and $b \in S V(0,+\infty)$. If $X=$ $L_{p, q ; b}\left(\mathbb{R}^{n}\right)$, then

$$
g_{\sigma} \in X^{\prime}
$$

if and only if either

$$
p>\frac{n}{\sigma}
$$

or

$$
p=\frac{n}{\sigma} \text { and }\left\|t^{-\frac{1}{q^{\prime}}}(b(t))^{-1}\right\|_{q^{\prime} ;(0,1)}<+\infty
$$

Proof By (56)

$$
\begin{aligned}
\left\|g_{\sigma}\right\|_{X^{\prime}} & \approx\left\|t^{\frac{1}{p^{\prime}}-\frac{1}{q^{\prime}}}(b(t))^{-1} g_{\sigma}^{*}(t)\right\|_{q^{\prime} ;(0,1)}+\left\|t^{\frac{1}{p^{\prime}}-\frac{1}{q^{\prime}}}(b(t))^{-1} g_{\sigma}^{*}(t)\right\|_{q^{\prime} ;(1,+\infty)} \\
& =: N_{1}+N_{2} .
\end{aligned}
$$

Since $\sigma \in(0, n)$, by (17),

$$
g_{\sigma}^{*}(t) \precsim t^{(\sigma-n) / n} e^{-c t^{1 / n}} \text { for all } t>0 .
$$

Due to the exponential factor involved in this estimate, we obtain $N_{2}<+\infty$. Thus, by (59),

$$
\left\|g_{\sigma}\right\|_{X^{\prime}}<+\infty \text { if and only if } N_{1}<+\infty .
$$


Using (12) and (15), we arrive at

$$
g_{\sigma}^{*}(t) \approx t^{(\sigma-n) / n} \text { for all } t \in(0,1) .
$$

This implies that $N_{1}<+\infty$ if and only if either

$$
\frac{1}{p^{\prime}}+\frac{\sigma}{n}-1=\frac{\sigma}{n}-\frac{1}{p}>0
$$

or

$$
\frac{1}{p^{\prime}}+\frac{\sigma}{n}-1=\frac{\sigma}{n}-\frac{1}{p}=0 \quad \text { and } \quad\left\|t^{-\frac{1}{q^{\prime}}}(b(t))^{-1}\right\|_{q^{\prime} ;(0,1)}<+\infty \text {. }
$$

To characterize (53) when $X$ is the Lorentz-Karamata space $L_{p, q ; b}\left(\mathbb{R}^{n}\right)$, we shall use the following lemma (with $\xi=1$ ).

Lemma 8 Let $\sigma \in(0,1), p \in\left[\frac{n}{\sigma},+\infty\right), q \in[1,+\infty], b \in S V(0,+\infty), r \in(0,+\infty]$, $\mu \in \mathcal{L}_{r}$ and let $\xi \in(0,1]$. Then

$$
\left\|t^{-\frac{1}{r}}(\mu(t))^{-1} \int_{0}^{t^{n}} \tau^{\frac{\sigma}{n}-1} f^{*}(\tau) d \tau\right\|_{r ;\left(0, \xi^{1 / n}\right)} \leq C_{1}\|f\|_{p, q ; b}
$$

for all $f \in L_{p, q ; b}\left(\mathbb{R}^{n}\right)$ if and only if

$$
\left\|t^{-\frac{1}{r}\left(\mu\left(t^{1 / n}\right)\right)^{-1}} \int_{0}^{t} h(\tau) d \tau\right\|_{r ;(0, \xi)} \leq C_{2}\left\|t^{\frac{1}{p}+\frac{1}{q^{\prime}}-\frac{\sigma}{n}} b(t) h(t)\right\|_{q ;(0, \xi)}
$$

for all $h \in \mathcal{M}^{+}(0, \xi)$. Moreover, the best possible constants $C_{1}=C_{1}(\xi)$ and $C_{2}=C_{2}(\xi)$ in (60) and (61) are equivalent and the constants involved in this equivalence are independent of $\xi$.

Proof (i) Assume that (60) holds. Then the inequality

$$
\left\|t^{-\frac{1}{r}}(\mu(t))^{-1} \int_{0}^{t^{n}} \tau^{\frac{\sigma}{n}-1} g(\tau) d \tau\right\|_{r ;\left(0, \xi^{1 / n}\right)} \precsim\left\|t^{\frac{1}{p}-\frac{1}{q}} b(t) g(t)\right\|_{q ;(0, \xi)}
$$

holds for all $g \in \mathcal{M}^{+}(0, \xi ; \downarrow)$. This implies that the inequality

$$
\begin{aligned}
\| t^{-\frac{1}{r}}(\mu(t))^{-1} & \int_{0}^{t^{n}} \tau^{\frac{\sigma}{n}-1}\left(\int_{\tau}^{\xi} h(s) s^{-\frac{\sigma}{n}} d s\right) d \tau \|_{r ;\left(0, \xi^{1 / n}\right)} \\
& \precsim\left\|t^{\frac{1}{p}-\frac{1}{q}} b(t) \int_{\tau}^{\xi} h(s) s^{-\frac{\sigma}{n}} d s\right\|_{q ;(0, \xi)}
\end{aligned}
$$

is satisfied for all $h \in \mathcal{M}^{+}(0, \xi)$. 
By Fubini's theorem,

$$
\begin{aligned}
\int_{0}^{t^{n}} \tau^{\frac{\sigma}{n}-1}\left(\int_{\tau}^{\xi} h(s) s^{-\frac{\sigma}{n}} d s\right) d \tau= & \int_{0}^{t^{n}} h(s) s^{-\frac{\sigma}{n}}\left(\int_{0}^{s} \tau^{\frac{\sigma}{n}-1} d \tau\right) d s \\
& +\int_{t^{n}}^{\xi} h(s) s^{-\frac{\sigma}{n}}\left(\int_{0}^{t^{n}} \tau^{\frac{\sigma}{n}-1} d \tau\right) d s \\
& \approx \int_{0}^{t^{n}} h(s) d s+t^{\sigma} \int_{t^{n}}^{\xi} h(s) s^{-\frac{\sigma}{n}} d s \\
\geq & \int_{0}^{t^{n}} h(s) d s \text { for all } t \in\left(0, \xi^{1 / n}\right)
\end{aligned}
$$

(the constants involved in this estimate are independent of $\xi$ ). This and a change of variables give

$$
\begin{aligned}
\operatorname{LHS}(63) & \succsim\left\|t^{-\frac{1}{r}}(\mu(t))^{-1} \int_{0}^{t^{n}} h(s) d s\right\|_{r ;\left(0, \xi^{1 / n}\right)} \\
& \approx\left\|t^{-\frac{1}{r}}\left(\mu\left(t^{1 / n}\right)\right)^{-1} \int_{0}^{t} h(s) d s\right\|_{r ;(0, \xi)} .
\end{aligned}
$$

On the other hand, the Hardy inequality (cf. Lemma 3)

$$
\left\|t^{\frac{1}{p}-\frac{1}{q}} b(t)\left(\int_{t}^{\xi} h(s) s^{-\frac{\sigma}{n}} d s\right)\right\|_{q ;(0, \xi)} \leq C\left\|t^{\frac{1}{p}+\frac{1}{q^{\prime}}-\frac{\sigma}{n}} b(t) h(t)\right\|_{q ;(0, \xi)}
$$

for all $h \in \mathcal{M}^{+}(0, \xi)$, with $C$ independent of $\xi$, shows that

$$
\operatorname{RHS}(63) \precsim\left\|t^{\frac{1}{p}+\frac{1}{q^{\prime}}-\frac{\sigma}{n}} b(t) h(t)\right\|_{q ;(0, \xi)} .
$$

The estimates (63)-(66) imply (61). The best possible constant $C_{2}$ in (61) satisfies $C_{2} \precsim C_{1}$.

(ii) Suppose now that (61) holds. Let $f \in L_{p, q ; b}\left(\mathbb{R}^{n}\right)$. Putting $h(t)=t^{\frac{\sigma}{n}-1} f^{*}(t)$, $t \in(0, \xi)$, and using a change of variables, we obtain

$$
\operatorname{LHS}(61) \approx\left\|t^{-\frac{1}{r}}(\mu(t))^{-1} \int_{0}^{t^{n}} \tau^{\frac{\sigma}{n}-1} f^{*}(\tau) d \tau\right\|_{r ;\left(0, \xi^{1 / n}\right)}=\operatorname{LHS}(60)
$$

and

$$
\begin{aligned}
\operatorname{RHS}(61) & \approx\left\|t^{\frac{1}{p}+\frac{1}{q^{\prime}}-\frac{\sigma}{n}} b(t) t^{\frac{\sigma}{n}-1} f^{*}(t)\right\|_{q ;(0, \xi)} \\
& =\left\|t^{\frac{1}{p}-\frac{1}{q}} b(t) f^{*}(t)\right\|_{q ;(0, \xi)} \precsim \operatorname{RHS}(60)
\end{aligned}
$$


(the constants involved in these estimates are independent of $\xi$ ). Therefore, (61) and estimates (67), (68) imply that (60) holds. The best possible constant $C_{1}$ in (60) satisfies $C_{1} \precsim C_{2}$.

Continuous embeddings of spaces $H^{\sigma} L_{p, q ; b}\left(\mathbb{R}^{n}\right)$ with $\sigma \in(0,1)$ into generalized Hölder spaces in the superlimiting case (that is, when $p>n / \sigma$ ) are characterized in the next theorem.

Theorem 3 Let $\sigma \in(0,1), p \in\left(\frac{n}{\sigma},+\infty\right), q \in[1,+\infty], b \in S V(0,+\infty), r \in(0,+\infty]$ and $\mu \in \mathcal{L}_{r}$. Let $\lambda:(0,1] \rightarrow(0,+\infty)$ be defined by

$$
\lambda(x):=x^{\sigma-\frac{n}{p}}\left(b\left(x^{n}\right)\right)^{-1} \text { for all } x \in(0,1] .
$$

(Note that $\lambda \in \mathcal{L}_{r}$ for any $\left.r \in(0,+\infty]\right)$.

(i) If $1 \leq q \leq r \leq+\infty$, then

$$
H^{\sigma} L_{p, q ; b}\left(\mathbb{R}^{n}\right) \hookrightarrow \Lambda_{\infty, r}^{\mu(\cdot)}\left(\overline{\mathbb{R}^{n}}\right)
$$

if and only if

$$
\varlimsup_{x \rightarrow 0_{+}} \frac{\lambda(x)}{\mu(x)}<+\infty .
$$

(ii) If $0<r<q \leq+\infty$ and $q>1$, then

$$
H^{\sigma} L_{p, q ; b}\left(\mathbb{R}^{n}\right) \hookrightarrow \Lambda_{\infty, r}^{\mu(\cdot)}\left(\overline{\mathbb{R}^{n}}\right)
$$

if and only if

$$
\int_{0}^{1}\left(\frac{\lambda(x)}{\mu(x)}\right)^{u} \frac{d x}{x}<+\infty
$$

where $\frac{1}{u}:=\frac{1}{r}-\frac{1}{q}$.

Proof Put $X=L_{p, q ; b}\left(\mathbb{R}^{n}\right)$. By Lemma $7,\left\|g_{\sigma}\right\|_{X^{\prime}}<+\infty$. Consequently, by Theorem 2 and Lemma 8 (with $\xi=1$ ),

$$
H^{\sigma} X \hookrightarrow \Lambda_{\infty, r}^{\mu(\cdot)}\left(\overline{\mathbb{R}^{n}}\right)
$$

if and only if, for all $h \in \mathcal{M}^{+}(0,1)$,

$$
\left\|t^{-\frac{1}{r}}\left(\mu\left(t^{1 / n}\right)\right)^{-1} \int_{0}^{t} h(\tau) d \tau\right\|_{r ;(0,1)} \precsim\left\|t^{\frac{1}{p}+\frac{1}{q^{\prime}}-\frac{\sigma}{n}} b(t) h(t)\right\|_{q ;(0,1) .}
$$

(i) If $1 \leq q \leq r \leq+\infty$, then Lemma 2 shows that (72) holds if and only if

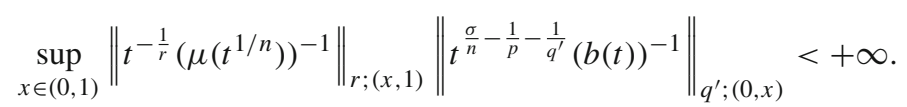

Since $\frac{\sigma}{n}-\frac{1}{p}>0$, Lemma 1(iii) implies that

$$
\left\|t^{\frac{\sigma}{n}-\frac{1}{p}-\frac{1}{q^{\prime}}}(b(t))^{-1}\right\|_{q^{\prime} ;(0, x)} \approx x^{\frac{\sigma}{n}-\frac{1}{p}}(b(x))^{-1} \text { for all } x \in(0,1) .
$$


Thus, (73) is equivalent to

$$
\sup _{x \in(0,1)}\left\|t^{-\frac{1}{r}}\left(\mu\left(t^{1 / n}\right)\right)^{-1}\right\|_{r ;(x, 1)} x^{\frac{\sigma}{n}-\frac{1}{p}}(b(x))^{-1}<+\infty .
$$

As $\mu$ and $b$ have singularities only at $0,(74)$ is satisfied if and only if

$$
\varlimsup_{x \rightarrow 0_{+}}\left\|t^{-\frac{1}{r}}\left(\mu\left(t^{1 / n}\right)\right)^{-1}\right\|_{r ;(x, 1)} x^{\frac{\sigma}{n}-\frac{1}{p}}(b(x))^{-1}<+\infty .
$$

Now, we are going to prove that (75) is equivalent to (70). First, suppose that (70) holds. Then there exists $\delta \in(0,1)$ such that

$$
\frac{1}{\mu\left(x^{1 / n}\right)} \precsim x^{\frac{1}{p}-\frac{\sigma}{n}} b(x) \text { for all } x \in(0, \delta) .
$$

On the other hand,

$$
\begin{aligned}
\left\|t^{-\frac{1}{r}}\left(\mu\left(t^{1 / n}\right)\right)^{-1}\right\|_{r ;(x, 1)} & \approx\left\|t^{-\frac{1}{r}}\left(\mu\left(t^{1 / n}\right)\right)^{-1}\right\|_{r ;(x, \delta)}+\left\|t^{-\frac{1}{r}}\left(\mu\left(t^{1 / n}\right)\right)^{-1}\right\|_{r ;(\delta, 1)} \\
& =: N_{1}+C(\delta),
\end{aligned}
$$

where $C(\delta)$ is a constant depending on $\delta$. Using (76) and the inequality $\frac{1}{p}-\frac{\sigma}{n}<0$, we arrive at

$$
N_{1} \precsim\left\|t^{-\frac{1}{r}} t^{\frac{1}{p}-\frac{\sigma}{n}} b(t)\right\|_{r ;(x, \delta)} \approx x^{\frac{1}{p}-\frac{\sigma}{n}} b(x)
$$

Thus,

$$
\left\|t^{-\frac{1}{r}}\left(\mu\left(t^{1 / n}\right)\right)^{-1}\right\|_{r ;(x, 1)} \precsim x^{\frac{1}{p}-\frac{\sigma}{n}} b(x)+C(\delta) \text { for all } x \in(0, \delta)
$$

and, using the properties of slowly varying functions, we obtain that

$$
\varlimsup_{x \rightarrow 0_{+}}\left\|t^{-\frac{1}{r}}\left(\mu\left(t^{1 / n}\right)\right)^{-1}\right\|_{r ;(x, 1)} x^{\frac{\sigma}{n}-\frac{1}{p}}(b(x))^{-1} \precsim \varlimsup_{x \rightarrow 0_{+}}\left(1+C(\delta) x^{\frac{\sigma}{n}-\frac{1}{p}}(b(x))^{-1}\right)=1,
$$

which gives (75).

Suppose now that (75) holds. Then there exists $\delta \in\left(0, \frac{1}{2}\right)$ such that, for all $x \in(0, \delta)$,

$$
\begin{aligned}
1 & \succsim\left\|t^{-\frac{1}{r}}\left(\mu\left(t^{1 / n}\right)\right)^{-1}\right\|_{r ;(x, 1)} x^{\frac{\sigma}{n}-\frac{1}{p}}(b(x))^{-1} \\
& \geq\left\|t^{-\frac{1}{r}}\left(\mu\left(t^{1 / n}\right)\right)^{-1}\right\|_{r ;(x, 2 x)} x^{\frac{\sigma}{n}-\frac{1}{p}}(b(x))^{-1} \\
& \geq\left(\mu\left((2 x)^{1 / n}\right)\right)^{-1}(\ln 2)^{1 / r} x^{\frac{\sigma}{n}-\frac{1}{p}}(b(x))^{-1} \\
& \approx\left(\mu\left((2 x)^{1 / n}\right)\right)^{-1}(2 x)^{\frac{\sigma}{n}-\frac{1}{p}}(b(2 x))^{-1},
\end{aligned}
$$

that is,

$$
\frac{y^{\frac{\sigma}{n}-\frac{1}{p}}(b(y))^{-1}}{\mu\left(y^{1 / n}\right)} \precsim 1 \text { for all } y \in(0,2 \delta)
$$

and (70) follows.

(ii) If $0<r<q \leq+\infty$ and $q>1$, then Lemma 2 states that (72) holds if and only if

$$
\int_{0}^{1}\left\|t^{-\frac{1}{r}}\left(\mu\left(t^{1 / n}\right)\right)^{-1}\right\|_{r ;(x, 1)}^{u}(V(x))^{u / r^{\prime}}\left(x^{\frac{\sigma}{n}-\frac{1}{p}}(b(x))^{-1}\right)^{q^{\prime}} \frac{d x}{x}<+\infty,
$$


where

$$
V(x)=\left\|t^{\frac{\sigma}{n}-\frac{1}{p}-\frac{1}{q^{\prime}}}(b(t))^{-1}\right\|_{q^{\prime} ;(0, x)}^{q^{\prime}}
$$

Since $\frac{\sigma}{n}-\frac{1}{p}>0$, Lemma 1(iii) and (69) imply that

$$
V(x) \approx\left(x^{\frac{\sigma}{n}-\frac{1}{p}}(b(x))^{-1}\right)^{q^{\prime}}=\left(\lambda\left(x^{1 / n}\right)\right)^{q^{\prime}}
$$

This and the identity $q^{\prime}\left(\frac{u}{r^{\prime}}+1\right)=u$ show that $(77)$ is equivalent to

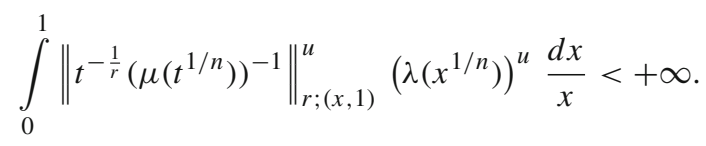

We claim that (79) and (71) are equivalent. Indeed, this follows on taking $\xi=1$ in the estimate

$$
\int_{0}^{\xi}\left\|t^{-\frac{1}{r}}\left(\mu\left(t^{1 / n}\right)\right)^{-1}\right\|_{r ;(x, \xi)}^{u}\left(\lambda\left(x^{1 / n}\right)\right)^{u} \frac{d x}{x} \approx \int_{0}^{\xi^{1 / n}}\left(\frac{\lambda(x)}{\mu(x)}\right)^{u} \frac{d x}{x}
$$

for all $\xi \in(0,1]$.

Thus, it remains to verify (80). Since $v:=\left(\frac{\sigma}{n}-\frac{1}{p}\right) r>0$ and $\frac{u}{r}>1,(69)$ and Lemma 3 imply that

$$
\begin{aligned}
\operatorname{LHS}(80) & =\left\|x^{-\frac{r}{u}}\left(\lambda\left(x^{1 / n}\right)\right)^{r} \int_{x}^{\xi} t^{-1}\left(\mu\left(t^{1 / n}\right)\right)^{-r} d t\right\|_{\frac{u}{r} ;(0, \xi)}^{\frac{u}{r}} \\
& =\left\|x^{\nu-\frac{r}{u}}(b(x))^{-r} \int_{x}^{\frac{u}{r}} t^{-1}\left(\mu\left(t^{1 / n}\right)\right)^{-r} d t\right\|_{\frac{u}{r} ;(0, \xi)} \\
& \precsim\left\|x^{\nu+1-\frac{r}{u}}(b(x))^{-r} x^{-1}\left(\mu\left(x^{1 / n}\right)\right)^{-r}\right\|_{\frac{u}{r} ;(0, \xi)}^{\frac{u}{r}} \\
& =\left\|x^{-\frac{r}{u}}\left(\lambda\left(x^{1 / n}\right)\right)^{r}\left(\mu\left(x^{1 / n}\right)\right)^{-r}\right\|_{\frac{u}{r} ;(0, \xi)}^{\frac{u}{r}} \\
& \approx \int_{0}^{1 / n}\left(\frac{\lambda(x)}{\mu(x)}\right)^{u} \frac{d x}{x} \text { for all } \xi \in(0,1] .
\end{aligned}
$$


Moreover,

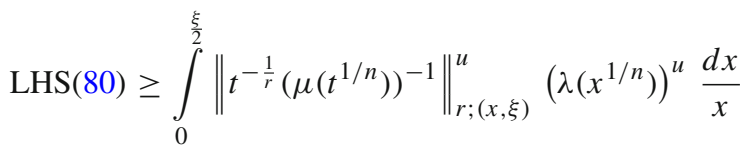

$$
\begin{aligned}
& \geq \int_{0}^{\frac{\xi}{2}}\left\|t^{-\frac{1}{r}}\left(\mu\left(t^{1 / n}\right)\right)^{-1}\right\|_{r ;(x, 2 x)}^{u}\left(\lambda\left(x^{1 / n}\right)\right)^{u} \frac{d x}{x} \\
& \geq \int_{0}^{\frac{\xi}{2}}\left(\mu\left((2 x)^{1 / n}\right)\right)^{-u}(\ln 2)^{u / r}\left(\lambda\left(x^{1 / n}\right)\right)^{u} \frac{d x}{x} \\
& \approx \int_{0}^{\xi^{1 / n}}\left(\frac{\lambda(x)}{\mu(x)}\right)^{u} \frac{d x}{x} \text { for all } \xi \in(0,1] .
\end{aligned}
$$

Combining estimates (81) and (82), we arrive at (80).

Remark 4 Assume that all the assumptions of Theorem 3 are satisfied.

(i) If $r \in[q,+\infty]$, then the embedding

$$
H^{\sigma} L_{p, q ; b}\left(\mathbb{R}^{n}\right) \hookrightarrow \Lambda_{\infty, r}^{\mu(\cdot)}\left(\overline{\mathbb{R}^{n}}\right)
$$

with $\mu=\lambda$ is sharp with respect to the parameter $\mu$ (which means that the target space $\Lambda_{\infty, r}^{\mu(\cdot)}\left(\overline{\mathbb{R}^{n}}\right)$ in (83) and the space $\Lambda_{\infty, r}^{\lambda(\cdot)}\left(\overline{\mathbb{R}^{n}}\right)$ (that is, the target space in (83) with $\mu=\lambda$ ) satisfy $\Lambda_{\infty, r}^{\lambda(\cdot)}\left(\overline{\mathbb{R}^{n}}\right) \hookrightarrow \Lambda_{\infty, r}^{\mu(\cdot)}\left(\overline{\mathbb{R}^{n}}\right)$ ). This follows from Theorem 3 (i) (condition (70)).

(ii) Among embeddings (83) that one with $\mu=\lambda$ and $r=q$ is optimal, that is, the target space $\Lambda_{\infty, r}^{\mu(\cdot)}\left(\overline{\mathbb{R}^{n}}\right)$ in $(83)$ and the space $\Lambda_{\infty, q}^{\lambda(\cdot)}\left(\overline{\mathbb{R}^{n}}\right)$ (that is, the target space in (83) with $\mu=\lambda$ and $r=q$ ) satisfy

$$
\Lambda_{\infty, q}^{\lambda(\cdot)}\left(\overline{\mathbb{R}^{n}}\right) \hookrightarrow \Lambda_{\infty, r}^{\mu(\cdot)}\left(\overline{\mathbb{R}^{n}}\right)
$$

Indeed, if $r \in[q,+\infty]$, this follows from part (i) and the fact that $\Lambda_{\infty, r}^{\lambda(\cdot)}\left(\overline{\mathbb{R}^{n}}\right) \hookrightarrow$ $\Lambda_{\infty, s}^{\lambda(\cdot)}\left(\overline{\mathbb{R}^{n}}\right)$ if $0<r<s \leq+\infty$ (cf. [22, Eq. (3.6)]). To verify it when $0<r<q$, note that (84) is satisfied if

$$
\left\|t^{-1 / r} \frac{\omega(f, t)}{\mu(t)}\right\|_{r ;(0,1)} \precsim\left\|t^{-1 / q} \frac{\omega(f, t)}{\lambda(t)}\right\|_{q ;(0,1)} \quad \text { for all } f \in \Lambda_{\infty, q}^{\lambda(\cdot)}\left(\overline{\mathbb{R}^{n}}\right) .
$$

Since $\omega(f, \cdot)$ is non-decreasing on $(0,1)$, this inequality holds (cf. [26, Proposition 2.1 (ii)] and [37, Lemma, p. 176]) if

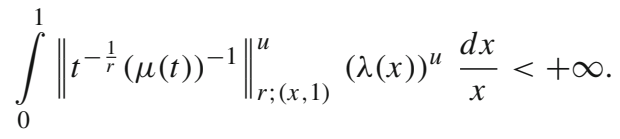

The last condition is equivalent to (71) (cf. the proof that (79) is equivalent to (71)). The result follows from Theorem 3 (ii) since (71) is satisfied when (83) holds. 
(iii) The embedding

$$
H^{\sigma} L_{p, q ; b}\left(\mathbb{R}^{n}\right) \hookrightarrow \Lambda_{\infty, r}^{\mu(\cdot)}\left(\overline{\mathbb{R}^{n}}\right)
$$

does not hold if $\mu=\lambda$ and $r \in(0, q)$ (this follows from Theorem 3 (ii)).

(iv) Using the terminology of [25] or [38], we obtain that $\left(\frac{\lambda(x)}{x}, q\right)$ is the continuity envelope of $H^{\sigma} L_{p, q ; b}\left(\mathbb{R}^{n}\right)$ (this is a consequence of part (i) with $r=+\infty$ and part (iii)).

The following assertion is an analogue of Theorem 3 and concerns the limiting case when $p=n / \sigma$.

Theorem 4 Let $\sigma \in(0,1), p=\frac{n}{\sigma}, q \in(1,+\infty], r \in(0,+\infty], \mu \in \mathcal{L}_{r}$ and let $b \in$ $S V(0,+\infty)$ be such that $\left\|t^{-\frac{1}{q^{\prime}}(b(t))^{-1}}\right\|_{q^{\prime} ;(0,1)}<+\infty$. Let $\lambda_{q r} \in \mathcal{L}_{r}$ be defined by

$$
\lambda_{q r}(x):=b^{q^{\prime} / r}\left(x^{n}\right)\left(\int_{0}^{x^{n}} b^{-q^{\prime}}(t) \frac{d t}{t}\right)^{\frac{1}{q^{\prime}+\frac{1}{r}}}, \quad x \in(0,1] .
$$

(i) If $1<q \leq r \leq+\infty$, then

$$
H^{\sigma} L_{p, q ; b}\left(\mathbb{R}^{n}\right) \hookrightarrow \Lambda_{\infty, r}^{\mu(\cdot)}\left(\overline{\mathbb{R}^{n}}\right)
$$

if and only if

$$
\varlimsup_{x \rightarrow 0_{+}} \frac{\left\|t^{-\frac{1}{r}}(\mu(t))^{-1}\right\|_{r ;(x, 1)}}{\left\|t^{-\frac{1}{r}}\left(\lambda_{q r}(t)\right)^{-1}\right\|_{r ;(x, 1)}}<+\infty .
$$

(ii) If $0<r<q \leq+\infty$ and $q>1$, then

$$
H^{\sigma} L_{p, q ; b}\left(\mathbb{R}^{n}\right) \hookrightarrow \Lambda_{\infty, r}^{\mu(\cdot)}\left(\overline{\mathbb{R}^{n}}\right)
$$

if and only if

$$
\int_{0}^{1 / 2}\left(\frac{\left\|t^{-1 / r}(\mu(t))^{-1}\right\|_{r ;(x, 1)}}{\left\|t^{-1 / r}\left(\lambda_{q r}(t)\right)^{-1}\right\|_{r ;(x, 1)}}\right)^{u}\left(\int_{0}^{x^{n}} t^{-1} b^{-q^{\prime}}(t) d t\right)^{-1} b^{-q^{\prime}}\left(x^{n}\right) \frac{d x}{x}<+\infty,
$$

where $\frac{1}{u}:=\frac{1}{r}-\frac{1}{q}$.

Proof Put $X=L_{p, q ; b}\left(\mathbb{R}^{n}\right)$. By Lemma $7,\left\|g_{\sigma}\right\|_{X^{\prime}}<+\infty$. Consequently, by Theorem 2 and Lemma 8 (with $\xi=1$ ),

$$
H^{\sigma} X \hookrightarrow \Lambda_{\infty, r}^{\mu(\cdot)}\left(\overline{\mathbb{R}^{n}}\right)
$$

if and only if, for all $h \in \mathcal{M}^{+}(0,1)$,

$$
\left\|t^{-\frac{1}{r}}\left(\mu\left(t^{1 / n}\right)\right)^{-1} \int_{0}^{t} h(\tau) d \tau\right\|_{r ;(0,1)} \precsim\left\|t^{\frac{1}{q^{\prime}}} b(t) h(t)\right\|_{q ;(0,1)} .
$$


(i) If $1<q \leq r \leq+\infty$, then Lemma 2 shows that (88) holds if and only if

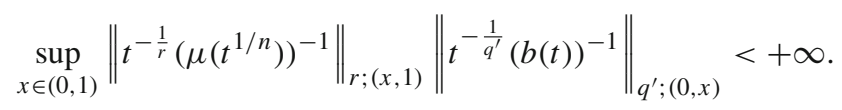

Since

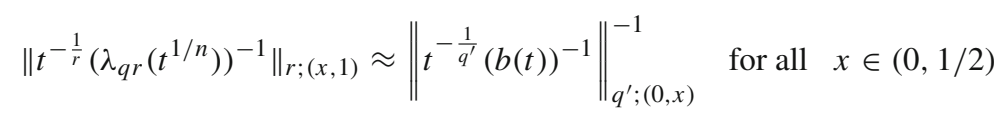

and as singularities of functions in question are only at $0,(89)$ is equivalent to

$$
\varlimsup_{x \rightarrow 0_{+}} \frac{\left\|t^{-\frac{1}{r}}\left(\mu\left(t^{1 / n}\right)\right)^{-1}\right\|_{r ;(x, 1)}}{\left\|t^{-\frac{1}{r}}\left(\lambda_{q r}\left(t^{1 / n}\right)\right)^{-1}\right\|_{r ;(x, 1)}}<+\infty
$$

and (86) follows.

(ii) If $0<r<q \leq+\infty$ and $q>1$, then Lemma 2 shows that (88) holds if and only if

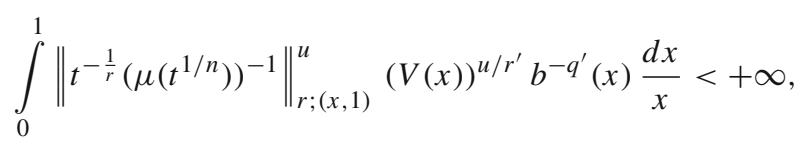

where

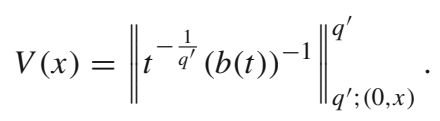

Using the identity $\frac{u}{r^{\prime}}=\frac{u}{q^{\prime}}-1,(90)$ and the fact that the singularities of the functions in question are only at 0 , we see that (91) is equivalent to (87).

Remark 5 Assume that all assumptions of Theorem 4 are satisfied.

(i) If $r \in[q,+\infty]$, then the embedding

$$
H^{\sigma} L_{p, q ; b}\left(\mathbb{R}^{n}\right) \hookrightarrow \Lambda_{\infty, r}^{\mu(\cdot)}\left(\overline{\mathbb{R}^{n}}\right)
$$

with $\mu=\lambda_{q r}$ is sharp with respect to the parameter $\mu$, that is, the target space $\Lambda_{\infty, r}^{\mu(\cdot)}\left(\overline{\mathbb{R}^{n}}\right)$ in (93) and the space $\Lambda_{\infty, r}^{\lambda_{q r}(\cdot)}\left(\overline{\mathbb{R}^{n}}\right)$ (that is, the target space in (93) with $\left.\mu=\lambda_{q r}\right)$ satisfy $\Lambda_{\infty, r}^{\lambda_{q r}(\cdot)}\left(\overline{\mathbb{R}^{n}}\right) \hookrightarrow \Lambda_{\infty, r}^{\mu(\cdot)}\left(\overline{\mathbb{R}^{n}}\right)$. Indeed, the last embedding is satisfied if

$$
\left\|t^{-1 / r} \frac{\omega(f, t)}{\mu(t)}\right\|_{r ;(0,1)} \precsim\left\|t^{-1 / r} \frac{\omega(f, t)}{\lambda_{q r}(t)}\right\|_{r ;(0,1)} \quad \text { for all } f \in \Lambda_{\infty, r}^{\lambda_{q r}(\cdot)}\left(\overline{\mathbb{R}^{n}}\right) .
$$

Since $\omega(f, \cdot)$ is non-decreasing on $(0,1)$, this inequality holds (cf. [26, Proposition 2.1 (i)]) if

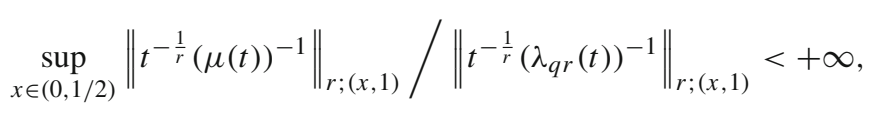

which is equivalent to (86). The result follows from Theorem 4 (i) since (86) is satisfied when (93) holds. 
(ii) Among embeddings (93) that one with $\mu=\lambda_{q q}$ and $r=q$ is optimal, that is, the target space $\Lambda_{\infty, r}^{\mu(\cdot)}\left(\overline{\mathbb{R}^{n}}\right)$ in (93) and the space $\Lambda_{\infty, q}^{\lambda_{q q}(\cdot)}\left(\overline{\mathbb{R}^{n}}\right)$ (that is, the target space in (93) with $\mu=\lambda_{q q}$ and $r=q$ ) satisfy

$$
\Lambda_{\infty, q}^{\lambda_{q q}(\cdot)}\left(\overline{\mathbb{R}^{n}}\right) \hookrightarrow \Lambda_{\infty, r}^{\mu(\cdot)}\left(\overline{\mathbb{R}^{n}}\right) .
$$

Indeed, if $r \in[q,+\infty]$, this follows from part (i) and the fact that $\Lambda_{\infty, r}^{\lambda_{q r}(\cdot)}\left(\overline{\mathbb{R}^{n}}\right) \hookrightarrow$ $\Lambda_{\infty, s}^{\lambda q s(\cdot)}\left(\overline{\mathbb{R}^{n}}\right)$ if $q \leq r \leq s \leq+\infty$ (the proof of this is analogous to the proof of [22, Eq. (3.18)]). To verify it when $0<r<q$, note that (94) is satisfied if

$$
\left\|t^{-1 / r} \frac{\omega(f, t)}{\mu(t)}\right\|_{r ;(0,1)} \precsim\left\|t^{-1 / q} \frac{\omega(f, t)}{\lambda_{q q}(t)}\right\|_{q ;(0,1)} \quad \text { for all } f \in \Lambda_{\infty, q}^{\lambda_{q q}(\cdot)}\left(\overline{\mathbb{R}^{n}}\right) .
$$

Since $\omega(f, \cdot)$ is non-decreasing on $(0,1)$, this inequality holds (cf. [26, Proposition 2.1 (ii)] and [37, Lemma, p. 176]) if

$$
\int_{0}^{1 / 2}\left\|t^{-\frac{1}{r}}(\mu(t))^{-1}\right\|_{r ;(x, 1)}^{u}\left\|t^{-\frac{1}{q}}\left(\lambda_{q q}(t)\right)^{-1}\right\|_{q ;(x, 1)}^{-q \frac{u}{r}}\left(\lambda_{q q}(x)\right)^{-q} \frac{d x}{x}<+\infty,
$$

which can be rewritten as (91). Furthermore, (91) is equivalent to (87) (cf. the proof of Theorem 4 (ii)). The result follows from Theorem 4 (ii) since (87) is satisfied when (93) holds.

(iii) The embedding

$$
H^{\sigma} L_{p, q ; b}\left(\mathbb{R}^{n}\right) \hookrightarrow \Lambda_{\infty, r}^{\mu(\cdot)}\left(\overline{\mathbb{R}^{n}}\right)
$$

does not hold if $\mu=\lambda_{q r}$ and $r \in(0, q)$ (this follows from Theorem 4 (ii)).

(iv) Using the terminology of [25] or [38], we obtain that $\left(\frac{\lambda_{q \infty}(x)}{x},+\infty\right)$ is the continuity envelope of $H^{\sigma} L_{p, q ; b}\left(\mathbb{R}^{n}\right)$ (this is a consequence of part (i) with $r=+\infty$ and part (iii)).

\section{Compactness}

First, we characterize totally bounded subsets of the space $\Lambda_{\infty, r}^{\mu(\cdot)}(\bar{\Omega})$.

Theorem 5 Let $r \in(0,+\infty), \mu \in \mathcal{L}_{r}$ and let $\Omega$ be a bounded domain in $\mathbb{R}^{n}$. Then $\mathcal{S} \subset$ $\Lambda_{\infty, r}^{\mu(\cdot)}(\bar{\Omega})$ is totally bounded if and only if $\mathcal{S}$ is bounded in $\Lambda_{\infty, r}^{\mu(\cdot)}(\bar{\Omega})$ and

$$
\sup _{u \in \mathcal{S}}\left\|t^{-\frac{1}{r}}(\mu(t))^{-1} \omega(u, t)\right\|_{r ;(0, \xi)} \rightarrow 0 \quad \text { as } \quad \xi \rightarrow 0_{+} .
$$

Proof SUFFICIENCY. Since $\mathcal{S}$ is bounded in $\Lambda_{\infty, r}^{\mu(\cdot)}(\bar{\Omega})$, Lemma 4 implies that $\mathcal{S}$ is also bounded in $C(\bar{\Omega})$.

Let $\varepsilon \in(0,1)$. By $(95)$, there is $\delta \in(0,1)$ such that

$$
\sup _{u \in \mathcal{S}}\left\|t^{-\frac{1}{r}}(\mu(t))^{-1} \omega(u, t)\right\|_{r ;(0, \delta)}<\frac{\varepsilon}{4} .
$$

Now, for this $\delta$, there is a positive constant $c(\delta)$ such that, for all $u \in \mathcal{S}$,

$$
\left\|u\left|\Lambda_{\infty, r}^{\mu(\cdot)}(\bar{\Omega})\|\leq c(\delta)\| u\right| L^{\infty}(\Omega)\right\|+\left\|t^{-\frac{1}{r}}(\mu(t))^{-1} \omega(u, t)\right\|_{r ;(0, \delta)} .
$$


By (96), for all $\xi$ with $|\xi|<\delta$,

$$
\begin{aligned}
1 & >\sup _{u \in \mathcal{S}}\left\|t^{-\frac{1}{r}}(\mu(t))^{-1} \omega(u, t)\right\|_{r ;(0, \xi)} \\
& =\sup _{u \in \mathcal{S}}\left\|t^{1-\frac{1}{r}}(\mu(t))^{-1} \widetilde{\omega}(u, t)\right\|_{r ;(0, \xi)} \\
& \succsim \sup _{u \in \mathcal{S}} \frac{\widetilde{\omega}(u, \xi)}{\mu(\xi)}\left\|t^{1-\frac{1}{r}}\right\|_{r ;(0, \xi)} \\
& \approx \sup _{u \in \mathcal{S}} \frac{\omega(u, \xi)}{\mu(\xi)} .
\end{aligned}
$$

Hence,

$$
\sup _{u \in \mathcal{S}} \omega(u, \xi) \precsim \mu(\xi), \quad|\xi|<\delta .
$$

Since $\mu(t) \rightarrow 0$ as $t \rightarrow 0_{+}$, we obtain that

$$
\sup _{u \in \mathcal{S}} \omega(u, \xi) \rightarrow 0 \text { as } \xi \rightarrow 0_{+},
$$

which means that $\mathcal{S}$ is equicontinuous. Therefore, the Ascoli-Arzelà theorem implies that $\mathcal{S}$ is totally bounded in $C(\bar{\Omega})$. Consequently, there exists a finite $\frac{\varepsilon}{2 c(\delta)}$-net $\left\{u_{1}, \ldots, u_{N}\right\} \subset \mathcal{S}$ such that

$$
\min _{k \in\{1, \ldots, N\}}\left\|u-u_{k} \mid L^{\infty}(\Omega)\right\|<\frac{\varepsilon}{2 c(\delta)} \quad \text { for all } \quad u \in \mathcal{S} .
$$

Using estimates (97), (98) and (96), we obtain for any $u \in \mathcal{S}$ that

$$
\begin{aligned}
\min _{k \in\{1, \ldots, N\}}\left\|u-u_{k} \mid \Lambda_{\infty, r}^{\mu(\cdot)}(\bar{\Omega})\right\| \leq & c(\delta) \min _{k \in\{1, \ldots, N\}}\left\|u-u_{k} \mid L^{\infty}(\Omega)\right\| \\
& +\sup _{k \in\{1, \ldots, N\}}\left\|t^{-\frac{1}{r}}(\mu(t))^{-1} \omega\left(u-u_{k}, t\right)\right\|_{r ;(0, \delta)} \\
\leq & c(\delta) \min _{k \in\{1, \ldots, N\}}\left\|u-u_{k} \mid L^{\infty}(\Omega)\right\| \\
& +\sup _{k \in\{1, \ldots, N\}}\left\|t^{-\frac{1}{r}}(\mu(t))^{-1} \omega(u, t)\right\|_{r ;(0, \delta)} \\
& +\sup _{k \in\{1, \ldots, N\}}\left\|t^{-\frac{1}{r}}(\mu(t))^{-1} \omega\left(u_{k}, t\right)\right\|_{r ;(0, \delta)} \\
< & \frac{\varepsilon}{2}+\frac{\varepsilon}{4}+\frac{\varepsilon}{4}=\varepsilon,
\end{aligned}
$$

which proves that $\mathcal{S}$ is totally bounded in $\Lambda_{\infty, r}^{\mu(\cdot)}(\bar{\Omega})$.

NECESSITY. Suppose that $\mathcal{S}$ is totally bounded in $\Lambda_{\infty, r}^{\mu(\cdot)}(\bar{\Omega})$. Then $\mathcal{S}$ is bounded in $\Lambda_{\infty, r}^{\mu(\cdot)}(\bar{\Omega})$. On the other hand, given $\varepsilon>0$, there exists a finite $\frac{\varepsilon}{2}$-net $\left\{u_{1}, \ldots, u_{N}\right\} \subset \mathcal{S}$ such that

$$
\min _{k \in\{1, \ldots, N\}}\left\|u-u_{k} \mid \Lambda_{\infty, r}^{\mu(\cdot)}(\bar{\Omega})\right\|<\frac{\varepsilon}{2} \text { for all } u \in \mathcal{S} .
$$

Because $r \in(0,+\infty)$, for each $k \in\{1, \ldots, N\}$ there is $\delta_{k}>0$ such that

$$
\left\|t^{-\frac{1}{r}}(\mu(t))^{-1} \omega\left(u_{k}, t\right)\right\|_{r ;\left(0, \delta_{k}\right)}<\frac{\varepsilon}{2} .
$$

Let $\delta:=\min _{k \in\{1, \ldots, N\}} \delta_{k}$. Since, for all $u \in \mathcal{S}$, any $k \in\{1, \ldots, N\}$ and all $t \in(0,1)$,

$$
\omega(u, t) \leq \omega\left(u-u_{k}, t\right)+\omega\left(u_{k}, t\right),
$$


(99) and (100) imply that

$$
\begin{aligned}
\left\|t^{-\frac{1}{r}}(\mu(t))^{-1} \omega(u, t)\right\|_{r ;(0, \delta) \leq} & \min _{k \in\{1, \ldots, N\}}\left\|t^{-\frac{1}{r}}(\mu(t))^{-1} \omega\left(u-u_{k}, t\right)\right\|_{r ;(0,1)} \\
& +\sup _{k \in\{1, \ldots, N\}}\left\|t^{-\frac{1}{r}}(\mu(t))^{-1} \omega\left(u_{k}, t\right)\right\|_{r ;(0, \delta)} \\
< & \frac{\varepsilon}{2}+\frac{\varepsilon}{2}=\varepsilon \text { for all } u \in \mathcal{S} .
\end{aligned}
$$

Therefore,

$$
\sup _{u \in \mathcal{S}}\left\|t^{-\frac{1}{r}}(\mu(t))^{-1} \omega(u, t)\right\|_{r ;(0, \delta)} \leq \varepsilon
$$

and (95) follows.

Remark 6 (i) In Theorem 5 the implication

$\mathcal{S} \subset \Lambda_{\infty, r}^{\mu(\cdot)}(\bar{\Omega})$ is bounded and (95) holds $\Rightarrow \mathcal{S}$ is totally bounded in $\Lambda_{\infty, r}^{\mu(\cdot)}(\bar{\Omega})$

remains true even if $r=+\infty$. (This can be seen from the proof of Theorem 5).

(ii) If $r=+\infty$ in Theorem 5, then the reverse implication to (101) holds provided that we assume $\mathcal{S} \subset \Lambda_{\infty, \infty}^{\mu(\cdot), 0}(\bar{\Omega})$. Here $\Lambda_{\infty, \infty}^{\mu(\cdot), 0}(\bar{\Omega})$ is a subspace of $\Lambda_{\infty, \infty}^{\mu(\cdot)}(\bar{\Omega})$ consisting of those functions $u$ which satisfy

$$
\lim _{\delta \rightarrow 0_{+}}\left\|(\mu(t))^{-1} \omega(u, t)\right\|_{\infty ;(0, \delta)}=0 .
$$

(This follows from the necessity part of the proof of Theorem 5).

(iii) Summarizing what we have said, we arrive at the following result.

Let $u \in \mathcal{L}$ and let $\Omega$ be a bounded domain in $\mathbb{R}^{n}$. Then $\mathcal{S} \subset \Lambda_{\infty, \infty}^{\mu(\cdot), 0}(\bar{\Omega})$ is totally bounded in $\Lambda_{\infty, \infty}^{\mu(\cdot)}(\bar{\Omega})$ if and only if $\mathcal{S}$ is bounded in $\Lambda_{\infty, \infty}^{\mu(\cdot)}(\bar{\Omega})$ and

$$
\sup _{u \in \mathcal{S}}\left\|(\mu(t))^{-1} \omega(u, t)\right\|_{\infty ;(0, \xi)} \rightarrow 0 \quad \text { as } \quad \xi \rightarrow 0_{+} .
$$

Now, we make use of Theorem 5 to characterize compact embeddings of Bessel potential spaces $H^{\sigma} X\left(\mathbb{R}^{n}\right)$ into generalized Hölder spaces $\Lambda_{\infty, r}^{\mu(\cdot)}(\bar{\Omega})$.

Theorem 6 Let $\sigma \in(0,1)$ and let $X=X\left(\mathbb{R}^{n}\right)=X\left(\mathbb{R}^{n}, \mu_{n}\right)$ be a r. i. BFS such that $\left\|g_{\sigma}\right\|_{X^{\prime}}<\infty$. Assume that $r \in(0,+\infty), \mu \in \mathcal{L}_{r}$ and that $\Omega$ is a bounded domain in $\mathbb{R}^{n}$. Then

$$
H^{\sigma} X\left(\mathbb{R}^{n}\right) \hookrightarrow \hookrightarrow \Lambda_{\infty, r}^{\mu(\cdot)}(\bar{\Omega})^{1}
$$

if and only if

$$
\sup _{\|f\|_{X} \leq 1}\left\|t^{-\frac{1}{r}}(\mu(t))^{-1} \int_{0}^{t^{n}} \tau^{\frac{\sigma}{n}-1} f^{*}(\tau) d \tau\right\|_{r ;(0, \xi)} \rightarrow 0 \quad \text { as } \quad \xi \rightarrow 0_{+} .
$$

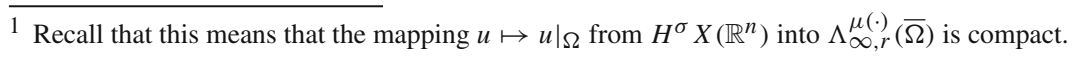


Proof SuffiCIENCY. By (103), there is $\delta \in(0,1)$ such that

$$
\sup _{\|f\|_{X} \leq 1}\left\|t^{-\frac{1}{r}}(\mu(t))^{-1} \int_{0}^{t^{n}} \tau^{\frac{\sigma}{n}-1} f^{*}(\tau) d \tau\right\|_{r ;(0, \delta)} \leq 1 .
$$

As, by Hölder inequality (cf. Remark 2),

$$
\int_{0}^{1} \tau^{\frac{\sigma}{n}-1} f^{*}(\tau) d \tau \precsim\left\|g_{\sigma}\right\|_{X^{\prime}}\|f\|_{X},
$$

and since the function $t \mapsto(\mu(t))^{-1}$ has a singularity only at 0 , we obtain for all $f \in X$ that

$$
\left\|t^{-\frac{1}{r}}(\mu(t))^{-1} \int_{0}^{t^{n}} \tau^{\frac{\sigma}{n}-1} f^{*}(\tau) d \tau\right\|_{r ;[\delta, 1)} \precsim\left\|t^{-\frac{1}{r}}(\mu(t))^{-1}\right\|_{r ;[\delta, 1)}\left\|g_{\sigma}\right\|_{X^{\prime}}\|f\|_{X} \precsim\|f\|_{X} .
$$

Together with estimate (104), this yields

$$
\sup _{\|f\|_{X} \leq 1}\left\|t^{-\frac{1}{r}(\mu(t))^{-1}} \int_{0}^{t^{n}} \tau^{\frac{\sigma}{n}-1} f^{*}(\tau) d \tau\right\|_{r ;(0,1)} \precsim 1
$$

Therefore, by Theorem $2, H^{\sigma} X\left(\mathbb{R}^{n}\right) \hookrightarrow \Lambda_{\infty, r}^{\mu(\cdot)}\left(\overline{\mathbb{R}^{n}}\right)$, which implies that the unit ball of $H^{\sigma} X$ is bounded in $\Lambda_{\infty, r}^{\mu(\cdot)}(\bar{\Omega})$.

Let $f \in X$ be such that $\|f\|_{X} \leq 1$. Then, by (48) of Remark 3,

$$
\begin{aligned}
\sup _{\|f\|_{X} \leq 1}\left\|t^{-\frac{1}{r}}(\mu(t))^{-1} \omega\left(f * g_{\sigma}, t\right)\right\|_{r ;(0, \xi)} & \\
& \precsim \sup _{\|f\|_{X} \leq 1}\left\|t^{-\frac{1}{r}}(\mu(t))^{-1} \int_{0}^{t^{n}} \tau^{\frac{\sigma}{n}-1} f^{*}(\tau) d \tau\right\|_{r ;(0, \xi)},
\end{aligned}
$$

which, together with (103), gives

$$
\sup _{\|f\|_{X} \leq 1}\left\|t^{-\frac{1}{r}}(\mu(t))^{-1} \omega\left(f * g_{\sigma}, t\right)\right\|_{r ;(0, \xi)} \rightarrow 0 \quad \text { as } \quad \xi \rightarrow 0_{+} .
$$

With respect to (18) and (19), this and Theorem 5 imply that the unit ball of the space $H^{\sigma} X$ is totally bounded in $\Lambda_{\infty, r}^{\mu(\cdot)}(\bar{\Omega})$ and the result follows.

NECESSITY. Suppose that (102) holds. Let $f \in X,\|f\|_{X} \leq 1$, and define $\bar{f}$ by (50) of Remark 3. Since $(\bar{f})^{*} \leq f^{*}$, we have that $\|\bar{f}\|_{X} \leq 1$. Moreover, by (49) of Remark 3, for all $\xi \in(0,1)$,

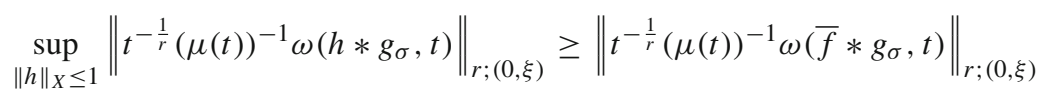

$$
\begin{aligned}
& \succsim\left\|t^{-\frac{1}{r}}(\mu(t))^{-1} \int_{0}^{t^{n}} \tau^{\frac{\sigma}{n}-1} f^{*}(\tau) d \tau\right\|_{r ;(0, \xi)} .
\end{aligned}
$$


Hence,

$$
\begin{aligned}
\sup _{\|h\|_{X} \leq 1}\left\|t^{-\frac{1}{r}}(\mu(t))^{-1} \omega\left(h * g_{\sigma}, t\right)\right\|_{r ;(0, \xi)} & \\
& \succsim \sup _{\|f\|_{X} \leq 1}\left\|t^{-\frac{1}{r}}(\mu(t))^{-1} \int_{0}^{t^{n}} \tau^{\frac{\sigma}{n}-1} f^{*}(\tau) d \tau\right\|_{r ;(0, \xi)},
\end{aligned}
$$

which, together with (102), (18), (19) and Theorem 5, gives (103).

Using Theorem 6 and Lemma 6, we arrive at the following corollary.

Corollary 3 Let $\sigma \in(0,1)$ and let $X=X\left(\mathbb{R}^{n}\right)$ be a r. i. BFS. Assume that $r \in(0,+\infty)$, $\mu \in \mathcal{L}_{r}$ and that $\Omega$ is a bounded domain in $\mathbb{R}^{n}$. Then (102) holds if and only if $\left\|g_{\sigma}\right\|_{X^{\prime}}<+\infty$ and (103) is satisfied.

Remark 7 (i) In Theorem 6 the implication (103) $\Longrightarrow$ (102) remains true even if $r=+\infty$. (This can be seen from Remark 6 (i) and the proof of Theorem 6.)

(ii) We see from Remark 6 (iii) that if we assume additionally in Theorem 6 that $r=+\infty$ and the space $X\left(\mathbb{R}^{n}\right)$ and $\mu \in \mathcal{L}$ are such that

$$
H^{\sigma} X\left(\mathbb{R}^{n}\right) \hookrightarrow \Lambda_{\infty, \infty}^{\mu(\cdot), 0}(\bar{\Omega}),
$$

then (102) is equivalent to (103).

(iii) For example, (105) is satisfied provided that

$$
\begin{aligned}
& \text { the Schwartz space } \mathscr{S}\left(\mathbb{R}^{n}\right) \text { is dense in } H^{\sigma} X\left(\mathbb{R}^{n}\right), \\
& \qquad \begin{array}{c}
H^{\sigma} X\left(\mathbb{R}^{n}\right) \hookrightarrow \Lambda_{\infty, \infty}^{\mu(\cdot)}(\bar{\Omega}), \\
\lim _{t \rightarrow 0_{+}} t / \mu(t)=0 .
\end{array}
\end{aligned}
$$

Indeed, given $u \in H^{\sigma} X\left(\mathbb{R}^{n}\right)$ and $\varepsilon>0$, there is $v \in \mathscr{S}\left(\mathbb{R}^{n}\right)$ such that $\|u-v\|_{H^{\sigma} X}<\varepsilon$. Moreover, $\omega(v, t) \leq c t$ for all $t \in(0,1)$, where $c=c(v)$ is a positive constant. Thus, using also (107), we obtain

$$
\begin{aligned}
\left\|(\mu(t))^{-1} \omega(u, t)\right\|_{\infty ;(0, \delta)} & \leq\left\|(\mu(t))^{-1} \omega(u-v, t)\right\|_{\infty ;(0, \delta)}+\left\|(\mu(t))^{-1} \omega(v, t)\right\|_{\infty ;(0, \delta)} \\
& \precsim\|u-v\|_{H^{\sigma} X}+c\|t / \mu(t)\|_{\infty ;(0, \delta)} \\
& \leq \varepsilon+c\|t / \mu(t)\|_{\infty ;(0, \delta)} \quad \text { for all } \delta \in(0,1) .
\end{aligned}
$$

Together with (108), this implies (105).

For instance, (106) holds if

the Schwartz space $\mathscr{S}\left(\mathbb{R}^{n}\right)$ is dense in $X\left(\mathbb{R}^{n}\right)$.

Indeed, this is a consequence of (18), (19), the fact that the mapping $h \mapsto g_{\sigma} * h$ maps $\mathscr{S}\left(\mathbb{R}^{n}\right)$ on $\mathscr{S}\left(\mathbb{R}^{n}\right)$, and (109).

In particular, (109) is satisfied provided that the r. i. BFS $X\left(\mathbb{R}^{n}\right)$ has absolutely continuous norm (cf. [13, Remark 3.13]). 


\section{Applications of the compactness result}

We are interested in compact embeddings in the case when the space $X$ is a Lorentz-Karamata space $L_{p, q ; b}\left(\mathbb{R}^{n}\right)$ with $p \in(1,+\infty), q \in[1,+\infty]$ and $b \in S V(0,+\infty)$. We shall start with the following result, which is a consequence of Lemma 8.

Lemma 9 Let $\sigma \in(0,1), p \in\left[\frac{n}{\sigma},+\infty\right), q \in[1,+\infty], b \in S V(0,+\infty), \xi \in(0,1)$, $r \in(0,+\infty]$ and let $\mu \in \mathcal{L}_{r}$. Then

$$
\sup _{\|f\|_{p, q ; b} \leq 1}\left\|t^{-\frac{1}{r}}(\mu(t))^{-1} \int_{0}^{t^{n}} \tau^{\frac{\sigma}{n}-1} f^{*}(\tau) d \tau\right\|_{r ;(0, \xi)} \rightarrow 0 \quad \text { as } \quad \xi \rightarrow 0_{+}
$$

if and only if

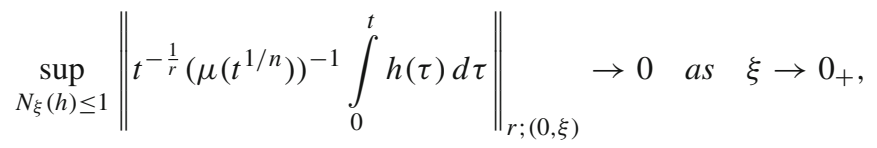

where $N_{\xi}(h):=\left\|t^{\frac{1}{p}+\frac{1}{q^{\prime}}-\frac{\sigma}{n}} b(t) h(t)\right\|_{q ;(0, \xi)}$ for all $h \in \mathcal{M}^{+}(0, \xi)$ and $\xi \in(0,1)$.

Compact embeddings of spaces $H^{\sigma} L_{p, q ; b}\left(\mathbb{R}^{n}\right)$ with $\sigma \in(0,1)$ into generalized Hölder spaces in the superlimiting case (that is, when $p>n / \sigma$ ) are characterized in the next theorem.

Theorem 7 Let $\sigma \in(0,1), p \in\left(\frac{n}{\sigma},+\infty\right), q \in[1,+\infty], b \in S V(0,+\infty), r \in(0,+\infty)$ and let $\mu \in \mathcal{L}_{r}$. Assume that $\Omega$ is a bounded domain in $\mathbb{R}^{n}$. Let $\lambda:(0,1] \rightarrow(0,+\infty)$ be defined by

$$
\lambda(x):=x^{\sigma-\frac{n}{p}}\left(b\left(x^{n}\right)\right)^{-1} \text { for all } x \in(0,1] .
$$

(Note that $\lambda \in \mathcal{L}_{r}$ for any $\left.r \in(0,+\infty]\right)$.

(i) If $1 \leq q \leq r<+\infty$, then

$$
H^{\sigma} L_{p, q ; b}\left(\mathbb{R}^{n}\right) \hookrightarrow \hookrightarrow \Lambda_{\infty, r}^{\mu(\cdot)}(\bar{\Omega})
$$

if and only if

$$
\lim _{x \rightarrow 0_{+}} \frac{\lambda(x)}{\mu(x)}=0 .
$$

(ii) If $0<r<q \leq+\infty$ and $q>1$, then

$$
H^{\sigma} L_{p, q ; b}\left(\mathbb{R}^{n}\right) \hookrightarrow \hookrightarrow \Lambda_{\infty, r}^{\mu(\cdot)}(\bar{\Omega})
$$

if and only if

$$
\int_{0}^{1}\left(\frac{\lambda(x)}{\mu(x)}\right)^{u} \frac{d x}{x}<+\infty
$$

where $\frac{1}{u}:=\frac{1}{r}-\frac{1}{q}$. 
Proof Put $X=L_{p, q ; b}\left(\mathbb{R}^{n}\right)$. By Lemma $7,\left\|g_{\sigma}\right\|_{X^{\prime}}<+\infty$. Consequently, by Theorem 6 and Lemma 9,

$$
H^{\sigma} X \hookrightarrow \hookrightarrow \Lambda_{\infty, r}^{\mu(\cdot)}(\bar{\Omega})
$$

if and only if

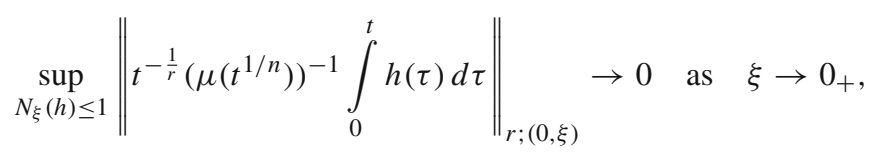

where $N_{\xi}(h):=\left\|t^{\frac{1}{p}+\frac{1}{q^{\prime}}-\frac{\sigma}{n}} b(t) h(t)\right\|_{q ;(0, \xi)}$ for all $h \in \mathcal{M}^{+}(0, \xi)$ and $\xi \in(0,1)$.

(i) If $1 \leq q \leq r<+\infty$, then Lemma 2 states that (115) holds if and only if

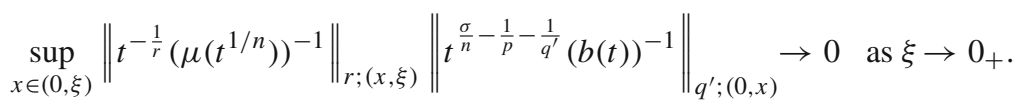

Since $\frac{\sigma}{n}-\frac{1}{p}>0$, Lemma 1(iii) shows that

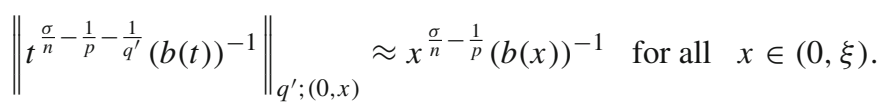

Thus, (116) is equivalent to

$$
\sup _{x \in(0, \xi)}\left\|t^{-\frac{1}{r}}\left(\mu\left(t^{1 / n}\right)\right)^{-1}\right\|_{r ;(x, \xi)} x^{\frac{\sigma}{n}-\frac{1}{p}}(b(x))^{-1} \rightarrow 0 \quad \text { as } \quad \xi \rightarrow 0_{+} .
$$

Now, we are going to prove that (117) is equivalent to (113). First, suppose that (113) holds. Then, given $\varepsilon>0$, there exists $\delta \in(0,1)$ such that

$$
\frac{x^{\frac{\sigma}{n}-\frac{1}{p}}(b(x))^{-1}}{\mu\left(x^{1 / n}\right)} \leq \varepsilon \text { for all } x \in(0, \delta) .
$$

Let $\xi \in(0, \delta)$. Using (118), the inequality $\frac{1}{p}-\frac{\sigma}{n}<0$ and Lemma 1(iii), we arrive at $\left\|t^{-\frac{1}{r}}\left(\mu\left(t^{1 / n}\right)\right)^{-1}\right\|_{r ;(x, \xi)} \leq \varepsilon\left\|t^{-\frac{1}{r}} t^{\frac{1}{p}-\frac{\sigma}{n}} b(t)\right\|_{r ;(x, 1)} \approx \varepsilon x^{\frac{1}{p}-\frac{\sigma}{n}} b(x)$ for all $x \in(0, \xi)$.

Consequently,

$$
\sup _{x \in(0, \xi)}\left\|t^{-\frac{1}{r}}\left(\mu\left(t^{1 / n}\right)\right)^{-1}\right\|_{r ;(x, \xi)} x^{\frac{\sigma}{n}-\frac{1}{p}}(b(x))^{-1} \precsim \varepsilon \quad \text { for all } \quad \xi \in(0, \delta),
$$

which gives (117).

Conversely, suppose that (117) holds. Then, given $\varepsilon>0$, there exists $\delta \in(0,1)$ such that, for all $\xi \in(0, \delta)$,

$$
\begin{aligned}
& \varepsilon \geq \sup _{x \in(0, \xi)}\left\|t^{-\frac{1}{r}}\left(\mu\left(t^{1 / n}\right)\right)^{-1}\right\|_{r ;(x, \xi)} x^{\frac{\sigma}{n}-\frac{1}{p}}(b(x))^{-1} \\
& \geq\left\|t^{-\frac{1}{r}}\left(\mu\left(t^{1 / n}\right)\right)^{-1}\right\|_{r ;(\xi / 2, \xi)}(\xi / 2)^{\frac{\sigma}{n}-\frac{1}{p}}(b(\xi / 2))^{-1} \\
& \geq\left(\mu\left(\xi^{1 / n}\right)\right)^{-1}(\ln 2)^{1 / r}(\xi / 2)^{\frac{\sigma}{n}-\frac{1}{p}}(b(\xi / 2))^{-1} \\
& \approx\left(\mu\left(\xi^{1 / n}\right)\right)^{-1} \xi^{\frac{\sigma}{n}-\frac{1}{p}}(b(\xi))^{-1},
\end{aligned}
$$


that is,

$$
\frac{\xi^{\frac{\sigma}{n}-\frac{1}{p}}(b(\xi))^{-1}}{\mu\left(\xi^{1 / n}\right)} \precsim \varepsilon \text { for all } \xi \in(0, \delta)
$$

and (113) follows.

(ii) If $0<r<q \leq+\infty$ and $q>1$, then Lemma 2 states that (115) holds if and only if

$$
\int_{0}^{\xi}\left\|t^{-\frac{1}{r}}\left(\mu\left(t^{1 / n}\right)\right)^{-1}\right\|_{r ;(x, \xi)}^{u}(V(x))^{u / r^{\prime}}\left(x^{\frac{\sigma}{n}-\frac{1}{p}}(b(x))^{-1}\right)^{q^{\prime}} \frac{d x}{x} \rightarrow 0 \text { as } \xi \rightarrow 0_{+},
$$

with $V$ from (78). Condition (119) can be rewritten as (cf. the proof that (77) is equivalent to (79))

$$
\int_{0}^{\xi}\left\|t^{-\frac{1}{r}}\left(\mu\left(t^{1 / n}\right)\right)^{-1}\right\|_{r ;(x, \xi)}^{u}\left(\lambda\left(x^{1 / n}\right)\right)^{u} \frac{d x}{x} \rightarrow 0 \text { as } \xi \rightarrow 0_{+} .
$$

Moreover, using (80), we see that (120) holds if and only if

$$
\int_{0}^{\xi^{1 / n}}\left(\frac{\lambda(x)}{\mu(x)}\right)^{u} \frac{d x}{x} \rightarrow 0 \text { as } \xi \rightarrow 0_{+}
$$

Finally, since the singularities of the functions $\lambda$ and $\mu$ are only at the origin, (121) is equivalent to (114).

The following assertion is an analogue of Theorem 7 and concerns the limiting case when $p=n / \sigma$.

Theorem 8 Let $\sigma \in(0,1), p=\frac{n}{\sigma}, q \in(1,+\infty], r \in(0,+\infty), \mu \in \mathcal{L}_{r}$ and let $b \in$ $S V(0,+\infty)$ be such that $\left\|t^{-\frac{1}{q^{\prime}}}(b(t))^{-1}\right\|_{q^{\prime} ;(0,1)}<+\infty$. Assume that $\Omega$ is a bounded domain in $\mathbb{R}^{n}$. Let $\lambda_{q r} \in \mathcal{L}_{r}$ be defined by

$$
\lambda_{q r}(x):=b^{q^{\prime} / r}\left(x^{n}\right)\left(\int_{0}^{x^{n}} b^{-q^{\prime}}(t) \frac{d t}{t}\right)^{\frac{1}{q^{\prime}+\frac{1}{r}}} \quad, \quad x \in(0,1] .
$$

(i) If $1<q \leq r<+\infty$, then

$$
H^{\sigma} L_{p, q ; b}\left(\mathbb{R}^{n}\right) \hookrightarrow \hookrightarrow \Lambda_{\infty, r}^{\mu(\cdot)}(\bar{\Omega})
$$

if and only if

$$
\lim _{x \rightarrow 0_{+}} \frac{\left\|t^{-\frac{1}{r}}(\mu(t))^{-1}\right\|_{r ;(x, 1)}}{\left\|t^{-\frac{1}{r}}\left(\lambda_{q r}(t)\right)^{-1}\right\|_{r ;(x, 1)}}=0 .
$$

(ii) If $0<r<q \leq+\infty$ and $q>1$, then

$$
H^{\sigma} L_{p, q ; b}\left(\mathbb{R}^{n}\right) \hookrightarrow \hookrightarrow \Lambda_{\infty, r}^{\mu(\cdot)}(\bar{\Omega})
$$


if and only if

$$
\int_{0}^{1 / 2}\left(\frac{\left\|t^{-1 / r}(\mu(t))^{-1}\right\|_{r ;(x, 1)}}{\left\|t^{-1 / r}\left(\lambda_{q r}(t)\right)^{-1}\right\|_{r ;(x, 1)}}\right)^{u}\left(\int_{0}^{x^{n}} t^{-1} b^{-q^{\prime}}(t) d t\right)^{-1} b^{-q^{\prime}}\left(x^{n}\right) \frac{d x}{x}<+\infty,
$$

where $\frac{1}{u}:=\frac{1}{r}-\frac{1}{q}$.

Proof Put $X=L_{p, q ; b}\left(\mathbb{R}^{n}\right)$. By Lemma $7,\left\|g_{\sigma}\right\|_{X^{\prime}}<+\infty$. Consequently, by Theorem 6 and Lemma 9,

$$
H^{\sigma} X \hookrightarrow \hookrightarrow \Lambda_{\infty, r}^{\mu(\cdot)}(\bar{\Omega})
$$

if and only if

$$
\sup _{N_{\xi}(h) \leq 1}\left\|t^{-\frac{1}{r}}\left(\mu\left(t^{1 / n}\right)\right)^{-1} \int_{0}^{t} h(\tau) d \tau\right\|_{r ;(0, \xi)} \rightarrow 0 \text { as } \xi \rightarrow 0_{+},
$$

where $N_{\xi}(h):=\left\|t^{\frac{1}{q^{\prime}}} b(t) h(t)\right\|_{q ;(0, \xi)}$ for all $h \in \mathcal{M}^{+}(0, \xi)$ and $\xi \in(0,1)$.

(i) If $1<q \leq r<+\infty$, by Lemma 2, (125) holds if and only if

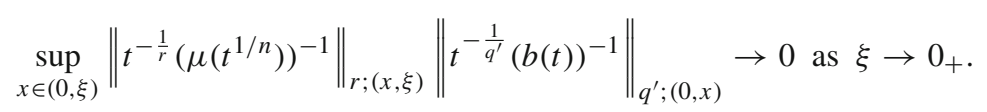

We show that (126) is equivalent to

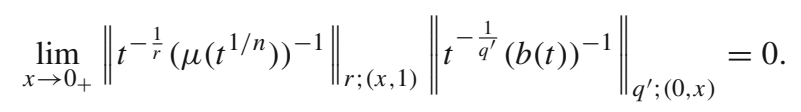

Indeed, assume that (126) holds. Then, given $\varepsilon>0$, there is $\Delta \in(0,1)$ such that

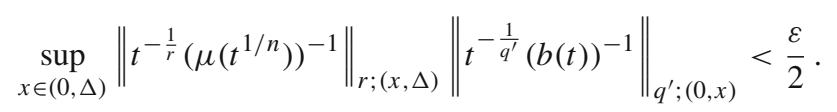

As $\left\|t^{-\frac{1}{q^{\prime}}}(b(t))^{-1}\right\|_{q^{\prime} ;(0,1)}<+\infty, q^{\prime}<+\infty$, and $\mu \in \mathcal{L}_{r}$, we can find $\delta \in(0, \Delta)$ such that

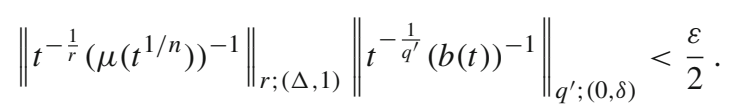

Therefore, for all $x \in(0, \delta)$,

$$
\begin{aligned}
& \left\|t^{-\frac{1}{r}}\left(\mu\left(t^{1 / n}\right)\right)^{-1}\right\|_{r ;(x, 1)}\left\|t^{-\frac{1}{q^{\prime}}(b(t))^{-1}}\right\|_{q^{\prime} ;(0, x)}
\end{aligned}
$$

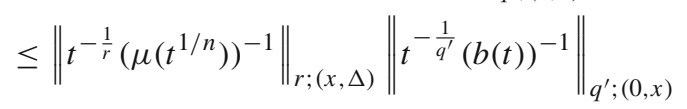

$$
\begin{aligned}
& +\left\|t^{-\frac{1}{r}}\left(\mu\left(t^{1 / n}\right)\right)^{-1}\right\|_{r ;(\Delta, 1)}\left\|t^{-\frac{1}{q^{\prime}}(b(t))^{-1}}\right\|_{q^{\prime} ;(0, x)} \\
& <\frac{\varepsilon}{2}+\frac{\varepsilon}{2}=\varepsilon,
\end{aligned}
$$


and (127) follows. The converse implication is a consequence of the estimate

$$
\begin{aligned}
& \sup _{x \in(0, \xi)}\left\|t^{-\frac{1}{r}}\left(\mu\left(t^{1 / n}\right)\right)^{-1}\right\|_{r ;(x, \xi)}\left\|t^{-\frac{1}{q^{\prime}}(b(t))^{-1}}\right\|_{q^{\prime} ;(0, x)}
\end{aligned}
$$

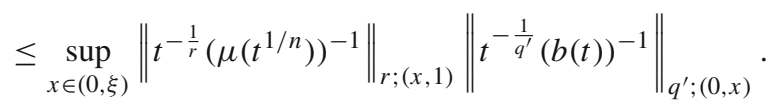

Since (90) holds, (127) is equivalent to (123) and the proof of part (i) is complete.

(ii) If $0<r<q \leq+\infty$ and $q>1$, then Lemma 2 shows that (125) holds if and only if

$$
\int_{0}^{\xi}\left\|t^{-\frac{1}{r}}\left(\mu\left(t^{1 / n}\right)\right)^{-1}\right\|_{r ;(x, \xi)}^{u}(V(x))^{u / r^{\prime}} b^{-q^{\prime}}(x) \frac{d x}{x} \rightarrow 0 \text { as } \xi \rightarrow 0_{+},
$$

with $V(x)$ from (92). Using the identity $\frac{u}{r^{\prime}}=\frac{u}{q^{\prime}}-1$ and (90), we see that (128) is equivalent to

$$
\int_{0}^{\xi} \frac{\left\|t^{-1 / r}(\mu(t))^{-1}\right\|_{r ;(x, \xi)}^{u}}{\left\|t^{-1 / r}\left(\lambda_{q r}(t)\right)^{-1}\right\|_{r ;(x, 1)}^{u}}\left(\int_{0}^{x^{n}} t^{-1} b^{-q^{\prime}}(t) d t\right)^{-1} b^{-q^{\prime}}\left(x^{n}\right) \frac{d x}{x} \rightarrow 0 \text { as } \xi \rightarrow 0_{+} .
$$

Finally, since singularities of functions in question are only at the origin, (129) is satisfied if and only if (124) holds.

Remark 8 (i) In Theorem 7 (i) the implication

$$
\Longrightarrow \quad H^{\sigma} L_{p, q ; b}\left(\mathbb{R}^{n}\right) \hookrightarrow \hookrightarrow \Lambda_{\infty, r}^{\mu(\cdot)}(\bar{\Omega})
$$

remains true even if we extend the range of $q$ and $r$ to $1 \leq q \leq r \leq+\infty$. (Indeed, this can be seen from the proof of Theorem 7 (i), where we use Theorem 6 and Remark 7 (i) instead of Theorem 6).

Theorem 7 (i) continues to hold if we assume that $1 \leq q \leq r \leq+\infty, q<+\infty$, and (108) is satisfied. (This follows from Remarks 7 (ii), (iii). Note that the condition $q<+\infty$ implies that the space $L_{p, q ; b}\left(\mathbb{R}^{n}\right)$ has absolutely continuous norm-cf. [33, Lemma 3.2].)

(ii) Similarly, in Theorem 8 (i) the implication

$$
\Longrightarrow \quad H^{\sigma} L_{p, q ; b}\left(\mathbb{R}^{n}\right) \hookrightarrow \hookrightarrow \Lambda_{\infty, r}^{\mu(\cdot)}(\bar{\Omega})
$$

remains true if we extend the range of $q$ and $r$ to $1<q \leq r \leq+\infty$.

Theorem 8 (i) continues to hold if we assume that $1<q \leq r \leq+\infty, q<+\infty$, and (108) is satisfied. (This follows from Remarks 7 (ii), (iii)).

Acknowledgments The work was supported by grant no. 201/05/2033 of the Grant Agency of the Czech Republic, by the Institutional Research Plan no. AV0Z10190503 of the Academy of Sciences of the Czech Republic (ASCR), by a joint project between ASCR and GRICES, by CIM and by Centre of Mathematics of the University of Coimbra.

\section{References}

1. Adams, R.A., Fournier, J.J.: Sobolev spaces. Pure and Applied Mathematics, vol. 140. Academic Press, Amsterdam (2003)

2. Aronszajn, N., Smith, K.: Theory of Bessel potentials. Part I, Ann. Inst. Fourier 11, 385-475 (1961) 
3. Aronszajn, N., Mulla, F., Szeptycki, P.: On spaces of potentials connected with $L^{p}$ classes. Part I. Ann. Inst. Fourier 13(2), 211-306 (1963)

4. Bennett, C., Rudnick, K.: On Lorentz-Zygmund spaces. Dissertationes Math. (Rozprawy Mat.) 175, 1-72 (1980)

5. Bennett, C., Sharpley, R.: Interpolation of operators. Pure and Applied Mathematics, vol. 129. Academic Press, New York (1988)

6. Bingham, N.H., Goldie, C.M., Teugels, J.L.: Regular Variation. Cambridge University Press, Cambridge (1987)

7. Brézis, H., Wainger, S.: A note on limiting cases of Sobolev embeddings. Comm. Partial Differ. Equ. 5, 773-789 (1980)

8. Calderón, A.P.: Lebesgue spaces of differentiable functions and distributions. In: Partial Differential Equations, Proc. Sympos. Pure Math., vol. 4, pp. 33-49. American Mathematical Society, Providence (1961)

9. DeVore, R.A., Lorentz, G.G.: Constructive Approximation. Grundlehren der mathematischen Wissenschaften-A series of Comprehensive Studies in Mathematics, vol. 303, Springer, Berlin (1993)

10. DeVore, R.A., Sharpley, R.C.: On the differentiability of functions in $R^{n}$. Proc. Amer. Math. Soc. 91, 326328 (1984)

11. Edmunds, D.E., Evans, W.D.: Hardy Operators, Function Spaces and Embeddings. Springer, Berlin (2004)

12. Edmunds, D.E., Gurka, P., Opic, B.: Double exponential integrability, Bessel potentials and embedding theorems. Studia Math. 115, 151-181 (1995)

13. Edmunds, D.E., Gurka, P., Opic, B.: On embeddings of logarithmic Bessel potential spaces. J. Funct. Anal. 146(1), 116-150 (1997)

14. Edmunds, D.E., Gurka, P., Opic, B.: Optimality of embeddings of logarithmic Bessel potential spaces. Q. J. Math. 51, 185-209 (2000)

15. Edmunds, D.E., Gurka, P., Opic, B.: Compact and continuous embeddings of logarithmic Bessel potential spaces. Studia Math. 168(3), 229-250 (2005)

16. Edmunds, D.E., Gurka, P., Opic, B.: Non-compact and sharp embeddings of logarithmic Bessel potential spaces into Hölder-type spaces. Z. Anal. Anwendungen 25, 73-80 (2006)

17. Edmunds, D.E., Kerman, R., Pick, L.: Optimal Sobolev imbeddings involving rearrangement-invariant quasinorms. J. Funct. Anal. 170, 307-355 (2000)

18. Edmunds, D.E., Triebel, H.: Logarithmic Sobolev spaces and their applications to spectral theory. Proc. Lond. Math. Soc. 71, 333-371 (1995)

19. Edmunds, D.E., Triebel, H.: Function Spaces, Entropy Numbers, Differential Operators. Cambridge University Press, Cambridge (1996)

20. Evans, W.D., Opic, B.: Real interpolation with logarithmic functors and reiteration. Canad. J. Math. 52, 920-960 (2000)

21. Gogatishvili, A., Neves, J.S., Opic, B.: Optimality of embeddings of Bessel-potential-type spaces into Lorentz-Karamata spaces. Proc. Roy. Soc. Edinburgh Sect. A 134, 1127-1147 (2004)

22. Gogatishvili, A., Neves, J.S., Opic, B.: Optimality of embeddings of Bessel-potential-type spaces into generalized Hölder spaces. Publ. Mat. 49, 297-327 (2005)

23. Gogatishvili, A., Neves, J.S., Opic, B.: Sharpness and non-compactness of embeddings of Besselpotential-type spaces. Math. Nachr. 280(9-10), 1083-1093 (2007)

24. Gogatishvili, A., Opic, B., Trebels, W.: Limiting reiteration for real interpolation with slowly varying functions. Math. Nachr. 278, 86-107 (2005)

25. Haroske, D.D.: Envelopes in function spaces—a first approach. Informatik Math/Inf/16/01. FriedrichSchiller, Universität Jena (2001)

26. Heinig, H.P., Stepanov, V.D.: Weighted Hardy inequalities for increasing functions. Canad. J. Math. 45, 104-116 (1993)

27. Marić, V.: Regular variation and differential equations. Lecture Notes in Mathematics, vol. 1726. Springer, Berlin (2000)

28. Maz'ja, V.G.: Sobolev Spaces. Springer, Berlin (1985)

29. Milman, M.: Extrapolation and optimal decompositions with applications to analysis. Lecture Notes in Mathematics, vol. 1580. Springer, Berlin (1994)

30. Neves, J.S.: Fractional Sobolev-type spaces and embeddings. PhD Thesis, University of Sussex (2001)

31. Neves, J.S.: Extrapolation results on general Besov-Hölder-Lipschitz spaces. Math. Nachr. 230, 117$141(2001)$

32. Neves, J.S.: Lorentz-Karamata spaces, Bessel and Riesz potentials and embeddings. Dissertationes Math. (Rozprawy Mat.) 405, 46 (2002) 
33. Neves, J.S.: Spaces of Bessel-potential type and embeddings: the super-limiting case. Math. Nachr. 265, 68-86 (2004)

34. Opic, B., Kufner, A.: Hardy-type inequalities. Pitman Research Notes in Math., Series 219. Longman Science and Technology, Harlow (1990)

35. Opic, B., Trebels, W.: Sharp embeddings of Bessel potential spaces with logarithmic smoothness. Math. Proc. Cambridge Philos. Soc. 134, 347-384 (2003)

36. Stein, E.M.: Singular Integrals and Differentiability Properties of Functions. Princeton University Press, Princeton (1970)

37. Stepanov, V.D.: The weighted Hardy's inequality for nonincreasing functions. Trans. Amer. Math. Soc. 338, 173-186 (1993)

38. Triebel, H.: The structure of functions. Monographs in Mathematics, vol. 97, Birkhäuser Verlag, Basel (2001)

39. Ziemer, W.: Weakly Differentiable Functions. Graduate Texts in Mathematics, vol. 120. Springer, Berlin (1989)

40. Zygmund, A.: Trigonometric Series, vol. I. Cambridge University Press, Cambridge (1957) 